\title{
Charged Black Holes in String Theory with Gauss-Bonnet Correction in Various Dimensions
}

\author{
Nobuyoshi Ohta ${ }^{1, *}$ and Takashi Torii ${ }^{2, \dagger}$ \\ ${ }^{1}$ Department of Physics, Kinki University, Higashi-Osaka, Osaka 577-8502, Japan \\ ${ }^{2}$ Department of General Education, Osaka Institute of Technology, Asahi-ku, Osaka 535-8585, Japan
}

(Dated: December 2, 2018)

\begin{abstract}
We study charged black hole solutions in Einstein-Gauss-Bonnet theory with the dilaton field which is the low-energy effective theory of the heterotic string. The spacetime is $D$-dimensional and assumed to be static and spherically symmetric with the $(D-2)$-dimensional constant curvature space and asymptotically flat. The system of the basic equations is complex and the solutions are obtained numerically. We identify the allowed parameter region where the black hole solutions exist, and show configurations of the field functions in $D=4-6$ and 10 . We also show the relations of the physical quantities of the black holes such as the horizon radius, the mass, the temperature, and so on, and find several results. The forms of the allowed parameter regions are different depending on the dimension. There is no extreme black hole solution with $T=0$ that can be obtained by taking the limit of the non-extreme solutions within the parameter range we chose. Entropy of the black holes in the dilatonic theory is always larger than that in the non-dilatonic theory. Our analysis includes the higher order term of the dilaton field which is not in our previous works. Its effect remarkably appears in five dimensions and is given in the appendix.

By our analysis it is found that the properties of the black hole solutions strongly depend on the dimension, charge, existence of the dilaton field. Hence both the detailed analyses of the individual systems and the investigations from the systematic point of view are important.
\end{abstract}

PACS numbers: 04.60.Cf, 04.50.Gh, 04.50.-h, 11.25.-w

\section{INTRODUCTION}

One of the most important problems in theoretical physics is to find the quantum theory of gravity and to apply it to physical systems to understand physics at strong gravity such as a black hole singularity. The leading candidates for that including all the fundamental forces of elementary particles are the ten-dimensional superstring theories or eleven-dimensional M-theory. The area where such quantum gravity plays the significant role includes the cosmology and black hole physics. There has been interest in applications of string/M theories to these subjects. Since it is still difficult to study geometrical settings in string/M theories directly, most analyses have been made by using low-energy effective theories inspired by string/M theories. The effective theories are the supergravities which typically involve not only the metric but also the dilaton field (as well as several gauge fields).

The first attempt at understanding black holes in the Einstein-Maxwell-dilaton system was made in Refs. [1, 2], in which a static spherically symmetric black hole solution with a dilaton hair was found. After that, many solutions were discussed in various models. On the other hand, it is known that there are correction terms of higher orders in the curvature to the lowest effective supergravity action coming from the superstring theories. The simplest correction is the Gauss-Bonnet (GB) term coupled to the dilaton field in the low-energy effective heterotic string [3]. It is then natural to ask how the black hole solutions are affected by the higher order terms in the effective theories.

When the dilaton field is dropped or is set to a constant by hand, the total action consists of the cosmological constant, Einstein-Hilbert and GB term, which are the first three terms in the Lovelock theory [4, 5]. Motivated by this observation, there have been many works on the black hole solutions in the Lovelock theory [6-9]. In the four-dimensional spacetime, the GB term does not give any contribution because it becomes a surface term and gives a topological invariant. Boulware and Deser (BD) [10] discovered a static, spherically symmetric black hole solutions of such models in more than four dimensions. In the system with a negative cosmological constant, black holes can have horizons with nonspherical topology such as torus, hyperboloid, and other compactified submanifolds. These solutions were originally found in general relativity and are called topological black holes [11]. Topological black hole solutions were studied also in the Einstein-GB (EGB) theory [12]. It is very interesting to see how these are modified

*Electronic address: ohtan@phys.kindai.ac.jp

†Electronic address: torii@ge.oit.ac.jp 
by the presence of a dilaton field. One of the purposes of this paper is to study charged black hole solutions with higher order corrections as well as the dilaton field.

There has been recently a renewed interest in these solutions as an application to the calculation of shear viscosity in the strongly coupled gauge theories using the black hole solutions in the five-dimensional EGB theory via AdS/CFT correspondence $[13,14]$. Almost all these studies considered the pure GB term without the dilaton field, or assumed a constant dilaton, which is not a solution of the heterotic string theory. It is, however, expected that AdS/CFT correspondence is valid within the effective theories of superstring which necessarily involve the dilaton field. The inclusion of the dilaton field was also considered by Boulware and Deser [10], but exact black hole solutions and their thermodynamic properties were not discussed. Callan et al. [15] considered black hole solutions in the theory with a higher-curvature term $R_{\mu \nu \rho \sigma} R^{\mu \nu \rho \sigma}$ and the dilaton field, and Refs. [8, 9] took both the GB term and the dilaton field into account in four-dimensional spacetime.

Hence it is important to study black hole solutions and their properties in the theory with both the higher order corrections and the dilaton field in general dimensions. In our earlier paper [16], we focused on asymptotically flat solutions and studied a system with the GB correction term and the dilaton field without the cosmological constant in various dimensions from 4 to 10 . They are spherically symmetric with the $(D-2)$-dimensional hypersurface of curvature signature $k=1$. We have then turned to planer symmetric $(k=0)$ topological black holes, but have found that no solution exists without the cosmological constant. In the string perspective, it is more interesting to examine asymptotically anti-de Sitter (AdS) black hole solutions with possible application to the AdS/CFT correspondence in mind. So in the sequel paper [17], we have presented the results on black hole solutions with a negative cosmological constant with $k=0$. In fact, shear viscosity has been computed using the result in that paper [14]. Other cases of solutions with $k= \pm 1$ and a negative cosmological term as well as topological black holes with and without the cosmological constant were studied in [18, 19], and their global structures were studied in Ref. [20]. Cosmological solutions were also considered in Ref. [21]. Other solutions are discussed in Refs. [22-29].

In the study of superconductors and superfluidity using the AdS/CFT correspondence, charged black hole solutions play important roles [30]. Hence it is also significant to extend our above studies of the neutral black hole solutions to charged ones. In particular, we should consider the inclusion of the dilaton field again.

There is another reason for our study of the system. In our above study of the neutral black holes [16-20], we did not consider the higher derivative term of the dilaton field, which also appears naturally as we will see below [3]. We intend to incorporate this term also and study how this modifies the solutions. As a result of our analysis, however, there is not much qualitative difference. Thus the results in our earlier papers should be useful.

This paper is organized as follows. In Sec. II, we first present the action and give basic equations to solve for the system of Einstein-Maxwell-Gauss-Bonnet term coupled to the dilaton field with a cosmological constant, although we will focus on the asymptotically flat solutions without cosmological constant later on. The case with the cosmological constant is left for future study. Boundary conditions and symmetry properties of the theory are also discussed in order to apply them in our following analysis. In Sec. III, we briefly summarize the thermodynamic properties of the solutions in the theory. In Sec. IV, we also briefly review the results for non-dilatonic solutions to see the difference from the dilatonic ones. We then proceed to the study of the charged black holes for $D=4,5,6$ and 10 in Secs. V, VI, VII and VIII, respectively. The study of the effects of the higher derivative of the dilaton field for the neutral black holes is delegated to the appendix, because we find that there is not much difference in the properties of the black hole solutions except for five dimensions. Some detailed discussions are given there. Section IX is devoted to conclusions and discussions.

\section{DILATONIC EINSTEIN-MAXWELL-GAUSS-BONNET THEORY}

\section{A. Action and basic equations}

We consider the following low-energy effective action for the heterotic string theory in one scheme [3]:

$$
\begin{aligned}
S= & \frac{1}{2 \kappa_{D}^{2}} \int d^{D} x \sqrt{-g} e^{-2 \phi}\left[R+4(\partial \phi)^{2}-\frac{1}{4} F^{2}-\frac{1}{12} H^{2}+\alpha_{2}\left\{R_{\mathrm{GB}}^{2}-16\left(R^{\mu \nu}-\frac{1}{2} R g^{\mu \nu}\right) \partial_{\mu} \phi \partial_{\nu} \phi\right.\right. \\
& +16 \square \phi(\partial \phi)^{2}-16(\partial \phi)^{4}-\frac{1}{2}\left(R^{\mu \nu \rho \sigma} H_{\mu \nu \alpha} H_{\rho \sigma}{ }^{\alpha}-2 R^{\mu \nu} H_{\mu \nu}^{2}+\frac{1}{3} R H^{2}\right)+2\left(D^{\mu} \partial^{\nu} \phi H_{\mu \nu}^{2}-\frac{1}{3} \mathbf{\square} \phi H^{2}\right) \\
& \left.\left.+\frac{2}{3} H^{2}(\partial \phi)^{2}+\frac{1}{24} H_{\mu \nu \lambda} H_{\rho \alpha}^{\nu} H^{\rho \sigma \lambda} H_{\sigma}{ }^{\mu \alpha}-\frac{1}{8} H_{\mu \nu}^{2} H^{2 \mu \nu}+\frac{1}{144}\left(H^{2}\right)^{2}\right\}\right],
\end{aligned}
$$


where $\kappa_{10}^{2}$ is a $D$-dimensional gravitational constant, $\phi$ a dilaton field, $F$ a gauge field strength, $H$ a 3 -form, $\alpha_{2}=\alpha^{\prime} / 8$ is a numerical coefficient given in terms of the Regge slope parameter $\alpha^{\prime}$, and $R_{\mathrm{GB}}^{2}=R_{\mu \nu \rho \sigma} R^{\mu \nu \rho \sigma}-4 R_{\mu \nu} R^{\mu \nu}+R^{2}$ is the GB term. $H^{2}=H^{\mu \nu \rho} H_{\mu \nu \rho}$ and $H_{\mu \nu}^{2}=H_{\mu}{ }^{\rho \sigma} H_{\nu \rho \sigma}$. In the original derivation of the effective action, it was first derived in the Einstein frame from the S-matrix calculation in the string theory, and then transformed into the string frame [3]. It is common and convenient to interpret results in the Einstein frame. Hence we transform Eq. (2.1) into the Einstein frame, reduce to $D$ dimensions, and use the field redefinition ambiguity $[3,31]$

$$
\begin{aligned}
\delta g_{\mu \nu} & =\alpha^{\prime}\left[a_{1} R_{\mu \nu}+a_{2} \nabla_{\mu} \phi \nabla_{\nu} \phi+g_{\mu \nu}\left\{a_{3} R+a_{4}(\nabla \phi)^{2}+a_{5} \nabla^{2} \phi\right\}\right], \\
\delta \phi & =\alpha^{\prime}\left[b_{1} R+b_{2}(\partial \phi)^{2}+b_{3} \nabla^{2} \phi\right],
\end{aligned}
$$

to obtain, up to higher order terms,

$$
S=\frac{1}{2 \kappa_{D}^{2}} \int d^{D} x \sqrt{-g}\left[R-\frac{1}{2}(\partial \phi)^{2}-\frac{1}{4} e^{-\gamma \phi} F^{2}+\alpha_{2} e^{-\gamma \phi}\left\{R_{\mathrm{GB}}^{2}+\frac{3}{16} a(\partial \phi)^{4}\right\}-\Lambda e^{\lambda \phi}\right]
$$

where $\gamma=1 / 2$. We have set $H=0$ because we focus on the effects of the dilaton field and the gauge field. Note that $H=0$ is a solution of the field equations.

When the action (2.4) is derived from (2.1), a numerical constant $a$ in the action (2.4) should be $a=1$. In our previous papers [16-20], however, we have examined the system with $a=0$, which corresponds to the case without the higher order term of the dilaton field. To see how the solutions are different with and without the higher order term of the dilaton field, we have included the constant $a$, and here we study mainly $a=1$ case. The difference between $a=0$ and $a=1$ is discussed in the Appendix. We have also included a cosmological constant with the dilaton coupling $\lambda$ although we study asymptotically flat solutions in this paper.

In the above process, higher order terms $\left(\geq O\left(\alpha^{\prime 2}\right)\right)$ are dropped. This is allowed because the effective low-energy action can be determined up to the field redefinition when it is read off from the scattering amplitudes computed in the string theories. Also note that the effective action was originally computed in the Einstein frame. All this means that there is no absolutely preferred form of the action if they are related up to terms of order $\alpha^{\prime 2}$. There might be some quantitative differences if we adopt theory of different choice of the coefficients, but we expect that the qualitative properties will not change significantly. It is the system (2.4) with $a=1$ that we study in this paper.

Let us consider the metric and field strength

$$
d s_{D}^{2}=-B(r) e^{-2 \delta(r)} d t^{2}+B(r)^{-1} d r^{2}+r^{2} h_{i j} d x^{i} d x^{j}, \quad F_{0 r}=\frac{d f(r)}{d r}
$$

where $h_{i j} d x^{i} d x^{j}$ represents the line element of a $(D-2)$-dimensional constant curvature space with curvature $(D-$ $2)(D-3) k$ and volume $\Sigma_{k}$ for $k= \pm 1,0$.

The field equations following from Eq. (2.4) are

$$
\begin{gathered}
{\left[(k-B) \tilde{r}^{D-3}\right]^{\prime} \frac{D-2}{\tilde{r}^{D-4}} h-\frac{1}{2} B \tilde{r}^{2} \phi^{\prime 2}-(D-1)_{4} e^{-\gamma \phi} \frac{(k-B)^{2}}{\tilde{r}^{2}}+4(D-2)_{3} \gamma e^{-\gamma \phi} B(k-B)\left(\phi^{\prime \prime}-\gamma \phi^{2}\right)} \\
+2(D-2)_{3} \gamma e^{-\gamma \phi} \phi^{\prime} \frac{(k-B)[(D-3) k-(D-1) B]}{\tilde{r}}-\frac{\tilde{r}^{2}}{2} e^{2 \delta-\gamma \phi} f^{\prime 2}+\frac{3}{16} a B^{2} \tilde{r}^{2} e^{-\gamma \phi} \phi^{\prime 4}-\tilde{r}^{2} \tilde{\Lambda} e^{\lambda \phi}=0 \\
\delta^{\prime}(D-2) \tilde{r} h+\frac{1}{2} \tilde{r}^{2} \phi^{\prime 2}-2(D-2)_{3} \gamma e^{-\gamma \phi}(k-B)\left(\phi^{\prime \prime}-\gamma \phi^{2}\right)-\frac{3}{8} a \tilde{r}^{2} B \phi^{\prime 4} e^{-\gamma \phi}=0 \\
\left(e^{-\delta} \tilde{r}^{D-2} B \phi^{\prime}\right)^{\prime}= \\
\gamma(D-2)_{3} e^{-\gamma \phi-\delta} \tilde{r}^{D-4}\left[(D-4)_{5} \frac{(k-B)^{2}}{\tilde{r}^{2}}+2\left(B^{\prime}-2 \delta^{\prime} B\right) B^{\prime}-4(k-B) B U(r)\right. \\
\left.-4 \frac{D-4}{\tilde{r}}\left(B^{\prime}-\delta^{\prime} B\right)(k-B)\right]+\gamma \tilde{r}^{D-2} e^{-\gamma \phi}\left(\frac{1}{2} e^{\delta} f^{\prime 2}+\frac{3}{16} a e^{-\delta} B^{2} \phi^{\prime 4}\right) \\
+\frac{3}{4} a \tilde{r}^{D-2} B \phi^{\prime 2} e^{-\delta-\gamma \phi}\left[3 B \phi^{\prime \prime}+\left\{2 B^{\prime}-\left(\delta^{\prime}+\gamma \phi^{\prime}-\frac{D-2}{r}\right) B\right\} \phi^{\prime}\right]+\tilde{r}^{D-2} \lambda \tilde{\Lambda} e^{-\delta+\lambda \phi}
\end{gathered}
$$


where we have defined the dimensionless variables: $\tilde{r}=r / \sqrt{\alpha_{2}}, \tilde{\Lambda}=\alpha_{2} \Lambda$, and the primes in the field equations denote the derivatives with respect to $\tilde{r}$. Namely we measure our length in the unit of $\sqrt{\alpha_{2}}$. In what follows, we omit tilde on the variables for simplicity. We have also defined

$$
\begin{aligned}
& (D-m)_{n}=(D-m)(D-m-1)(D-m-2) \cdots(D-n), \\
& h=1+2(D-3) e^{-\gamma \phi}\left[(D-4) \frac{k-B}{r^{2}}+\gamma \phi^{\prime} \frac{3 B-k}{r}\right], \\
& \tilde{h}=1+2(D-3) e^{-\gamma \phi}\left[(D-4) \frac{k-B}{r^{2}}+\gamma \phi^{\prime} \frac{2 B}{r}\right] \text {, } \\
& U(r)=(2 \tilde{h})^{-1}\left[(D-3)_{4} \frac{k-B}{r^{2} B}-2 \frac{D-3}{r}\left(\frac{B^{\prime}}{B}-\delta^{\prime}\right)-\frac{1}{2} \phi^{\prime 2}\right. \\
& +(D-3) e^{-\gamma \phi}\left\{(D-4)_{6} \frac{(k-B)^{2}}{r^{4} B}-4(D-4)_{5} \frac{k-B}{r^{3}}\left(\frac{B^{\prime}}{B}-\delta^{\prime}-\gamma \phi^{\prime}\right)\right. \\
& -4(D-4) \gamma \frac{k-B}{r^{2}}\left(\gamma \phi^{\prime 2}+\frac{D-2}{r} \phi^{\prime}-\Phi\right)+8 \frac{\gamma \phi^{\prime}}{r}\left[\left(\frac{B^{\prime}}{2}-\delta^{\prime} B\right)\left(\gamma \phi^{\prime}-\delta^{\prime}+\frac{2}{r}\right)\right. \\
& \left.\left.-\frac{D-4}{2 r} B^{\prime}\right]+4(D-4)\left(\frac{B^{\prime}}{2 B}-\delta^{\prime}\right) \frac{B^{\prime}}{r^{2}}-\frac{4 \gamma}{r} \Phi\left(B^{\prime}-2 \delta^{\prime} B\right)\right\}+\frac{1}{2 B} e^{2 \delta-\gamma \phi} f^{\prime 2} \\
& \left.+\frac{3}{16} a B \phi^{\prime 4} e^{-\gamma \phi}-\frac{1}{B} \Lambda e^{\lambda \phi}\right] \\
& \Phi=\phi^{\prime \prime}+\left(\frac{B^{\prime}}{B}-\delta^{\prime}+\frac{D-2}{r}\right) \phi^{\prime} .
\end{aligned}
$$

The field equation for the Maxwell field (2.9) is easily integrated to give

$$
f^{\prime}=\frac{q}{r^{D-2}} e^{\gamma \phi-\delta}
$$

where $q$ is a constant corresponding to the charge. It should be noted that the quantity $q$ in this equation is actually the rescaled one $\tilde{q}=q / \alpha^{(D-3) / 2}$ related to the real charge $q$. But as we mentioned before, tilde is omitted here and below.

Our task is reduced to setting boundary conditions for the metric functions $B, \delta$ and the dilaton field $\phi$ and integrate the above set of equations, just like in our previous papers [16-20].

\section{B. Boundary conditions and asymptotic behaviors}

In this paper we consider non-extreme solutions which has a non-degenerate black hole horizon. Hence at the horizon $r_{H}$,

$$
B_{H}=0, \quad B_{H}^{\prime} \neq 0,
$$

where $B_{H}=B\left(r_{H}\right)$. Here and in what follows, quantities evaluated at the horizon are represented by a subscript $H$. At the horizon, Eq. (2.6) gives

$$
\begin{aligned}
h_{H} B_{H}^{\prime} & =\frac{(D-3) k}{r_{H}}+k^{2}(D-3)_{5} \frac{e^{-\gamma \phi_{H}}}{r_{H}^{3}}-\frac{q^{2}}{2(D-2)} \frac{e^{\gamma \phi_{H}}}{r_{H}^{2 D-5}}-\frac{r_{H} \Lambda}{D-2} e^{\lambda \phi_{H}}, \\
& =\frac{(D-3) k}{r_{H}}+k^{2}(D-4)_{5} \frac{C}{2 r_{H}}-\frac{D-3}{D-2} \frac{q^{2}}{r_{H}^{2 D-3} C}-\frac{r_{H} \Lambda}{D-2} e^{\lambda \phi_{H}},
\end{aligned}
$$

where

$$
C=\frac{2(D-3) e^{-\gamma \phi_{H}}}{r_{H}^{2}}
$$


Henceforth we restrict our consideration to $k=1$ and $\Lambda=0$ case. Combining Eq. (2.16) with Eq. (2.8) evaluated at the horizon, we obtain the quadratic equation determining $\phi_{H}^{\prime}$ :

$$
A_{2} \phi_{H}^{\prime 2}+A_{1} \phi_{H}^{\prime}+A_{0}=0
$$

where

$$
\begin{aligned}
A_{2}= & C \gamma r_{H}^{2 D+2}\left[-2(D-3)\left\{C\left((D-2) \gamma^{2}[2 C(D-3)-1]+D-4\right)+1\right\} q^{2} r_{H}^{4}\right. \\
& \left.+C(D-2) r_{H}^{2 D}\left\{C(D-4)\left(C(D-2) \gamma^{2}[2 C(D-5)(D-3)+3 D-11]+C(D-4)_{5}+3 D-11\right)+2(D-3)\right\}\right] \\
A_{1}= & -C(D-2) r_{H}^{4 D+1}\left\{C^{2}(D-4)(D-1){ }_{2} \gamma^{2}\left[(D-4)_{5} C^{2}-2 C-2\right]+\left[C(D-4)_{5}+2(D-3)\right][C(D-4)+1]^{2}\right\} \\
& +2(D-3) q^{2} r_{H}^{2 D+5}\left\{2 C(C-1)(D-2) \gamma^{2}[2 C(D-4)+1]+[C(D-4)+1]^{2}\right\}+4 C(D-3)^{2} q^{4} r_{H}^{9} \gamma^{2}(2.20) \\
A_{0}= & -\frac{1}{2} C^{2}(D-2)^{2}(D-1) \gamma\{C(D-4)[C(D-4)(D+1)+4]-2(D-2)\} r_{H}^{4 D}+2(D-3)^{2} q^{4} r_{H}^{8} \gamma \\
& -2(D-2)_{3} q^{2} \gamma\left[3(D-4) C^{2}+6 C-1\right] r_{H}^{2 D+4}
\end{aligned}
$$

The $(\partial \phi)^{4}$ term in Eq. (2.4) does not contribute to the boundary condition of the dilaton field at the horizon.

In the asymptotic region of $r \rightarrow \infty$, we assume

$$
\begin{aligned}
B & \rightarrow 1-\frac{2 M}{r^{D-3}}+\cdots \\
\delta & \rightarrow 0 \\
\phi & \rightarrow 0
\end{aligned}
$$

where $M$ is a constant corresponding to the mass of the black hole. Although $\delta \rightarrow \delta_{\infty}$ and $\phi \rightarrow \phi_{\infty}$, where $\delta_{\infty}$ and $\phi_{\infty}$ are constant, these constants can be rescaled out to satisfy Eqs. (2.23) and (2.24) by the symmetries shown below, and the solutions are asymptotically flat.

\section{Symmetry and scaling}

It is useful to consider several symmetries of our field equations (or our model).

First, our field equations (2.6) - (2.8) have a shift symmetry:

$$
\phi \rightarrow \phi-\phi_{*}, \quad r \rightarrow e^{\gamma \phi_{*} / 2} r, \quad q \rightarrow e^{(D-2) \gamma \phi_{*} / 2} q, \quad\left(\Lambda \rightarrow e^{(\lambda-\gamma) \phi_{*}} \Lambda\right),
$$

where $\phi_{*}$ is an arbitrary constant. ${ }^{1}$ This changes the magnitude of the cosmological constant when we consider black hole solutions in its presence. Hence this may be used to generate solutions for different values of the cosmological constant, given a solution with some value of cosmological constant. Even without the cosmological constant, this symmetry can be used to change the asymptotic value of the dilaton field.

The second one is another shift symmetry under

$$
\delta \rightarrow \delta-\delta_{*}, \quad t \rightarrow e^{-\delta_{*}} t
$$

with an arbitrary constant $\delta_{*}$, which may be used to shift the asymptotic value of $\delta$ to zero. ${ }^{2}$

\footnotetext{
1 There are typos in our previous papers $[18,19]$ in the exponent of the transformation rule in the second term; they should have the + sign.

${ }^{2}$ For $k=0$, there is another symmetry. The field equations (2.6)-(2.8) are invariant under the scaling transformation $B \rightarrow c^{2} B, r \rightarrow c r$, with an arbitrary constant $c$. If a black hole solution with the horizon radius $r_{H}$ is obtained, we can generate solutions with different horizon radii but the same $\Lambda$ by this scaling transformation.
} 


\section{THERMODYNAMICAL VARIABLES}

Here we briefly summarize thermodynamical quantities of black holes to be used in the following discussions. The Hawking temperature is given by the periodicity of the Euclidean time on the horizon as (keeping $k$ arbitrary)

$$
\begin{aligned}
T_{H} & =\frac{e^{-\delta_{H}}}{4 \pi} B_{H}^{\prime} \\
& =\frac{e^{-\delta_{H}}}{4 \pi h_{H}}\left[\frac{(D-3) k}{r_{H}}+\frac{(D-3)_{5} k^{2}}{r_{H}^{3}} e^{-\gamma \phi_{H}}-\frac{q^{2}}{2(D-2)} \frac{e^{\gamma \phi_{H}}}{r_{H}^{2 D-5}}\right] .
\end{aligned}
$$

Along the definition of entropy in Ref. [32], which originates from the Noether charge associated with the diffeomorphism invariance of the system, we obtain

$$
S=-2 \pi \int_{\Sigma} \frac{\partial \mathcal{L}}{\partial R_{\mu \nu \rho \sigma}} \epsilon_{\mu \nu} \epsilon_{\rho \sigma}
$$

where $\Sigma$ is the event horizon $(D-2)$-surface, $\mathcal{L}$ is the Lagrangian density, $\epsilon_{\mu \nu}$ denotes the volume element binormal to $\Sigma$. This entropy has desirable properties such that it obeys the first law of black hole thermodynamics and that it is expected to obey even the second law [33]. For our present model, this gives

$$
S=\frac{r_{H}^{D-2} \Sigma_{k}}{4}\left[1+2(D-2)_{3} \frac{k e^{-\gamma \phi_{H}}}{r_{H}^{2}}\right]-S_{\min } .
$$

It is noted again that $\Sigma_{k}$ is the volume of the unit constant curvature space, and $\Sigma_{1}=\frac{2 \pi^{(D-1) / 2}}{\Gamma((D-1) / 2)}$. $S_{\min }$ is added to make the entropy non-negative [34].

\section{NON-DILATONIC BLACK HOLE SOLUTION}

It will be instructive to compare our results with the non-dilatonic case. When the dilaton field is absent (i.e., Einstein-Maxwell-GB system), we substitute $\phi \equiv 0$ and $\gamma=0$ into Eqs. (2.6) and (2.7). In the $D=4$ case, the GB term is total divergence and does not give any contribution to the field equations. As a result, the solution reduces to the Reissner-Nordström (RN) solution.

For $D \geq 5$, the field equations can be integrated to yield [22]

$$
\begin{gathered}
\bar{B}=1-\frac{2 \bar{m}}{r^{D-3}}, \\
\delta=0,
\end{gathered}
$$

where

$$
\bar{m}=\frac{r^{D-1}}{4(D-3)_{4}}\left[-1 \pm \sqrt{1+\frac{8(D-3)_{4} \bar{M}}{r^{D-1}}-\frac{(D-4) q^{2}}{8(D-2) r^{2(D-2)}}}\right]
$$

and $\bar{M}$ is an integration constant corresponding to the asymptotic value $\bar{m}(\infty)$ for the plus sign in Eq. (4.3). In the $\alpha_{2} \rightarrow 0$ limit, the solutions with the plus sign approach the RN solutions. This means that they can be considered to be the solution with GB correction to general relativity (GR). On the other hand, the solutions with the minus sign do not have such a limit. For these reasons, we call the solutions with plus (minus) sign the (non-)GR branch.

For $\bar{M}=0$ and $q=0$, the metric function becomes

$$
\bar{B}= \begin{cases}1 & (\text { GR branch }) \\ 1+\frac{r^{2}}{\ell_{\text {eff }}^{2}} & (\text { non-GR branch })\end{cases}
$$

where $\ell_{\mathrm{eff}}^{2}=(D-3)_{4}$. Hence the spacetime is Minkowski in the GR branch while the spacetime is anti-de Sitter in the non-GR branch although the cosmological constant $\Lambda$ is absent. 
For the charged solution, besides the central singularity at $r=0$, there can be another known as the branch singularity at finite radius $r_{b}>0$, which is obtained by the condition that the inside of the square root in Eq. (4.3) vanishes. We find the $\bar{M}-r_{b}$ relation

$$
\bar{M}=\frac{r_{b}^{D-1}}{8(D-3)_{4}}\left[\frac{(D-4) q^{2}}{8(D-2) r_{b}^{2(D-2)}}-1\right]
$$

This implies that the branch singularity can appear for positive mass in charged black hole, but only for negative mass parameter in the neutral black hole.

It can be shown that there is no black hole solution in the non-GR branch. On the other hand, in the GR branch, Eq. (4.3) evaluated at the horizon $\bar{B}=0$ gives

$$
\bar{M}=\frac{1}{2} r_{H}^{D-5}\left[r_{H}^{2}+(D-3)_{4}+\frac{q^{2}}{8(D-2)_{3} r_{H}^{2 D}}\right] .
$$

This is the $\bar{M}-r_{H}$ relation for the black hole without the dilaton field. For $q=0$, we see that $D=5$ is special because only in this case the mass goes to a finite value in the limit of $r_{H} \rightarrow 0$, whereas it vanishes for other dimensions including four. This limit does not exist for charged ones $(q \neq 0)$.

\section{V. $D=4$ BLACK HOLE}

For $D=4$, Eq. (2.18) to determine $\phi_{H}^{\prime}$ reduces to

$$
\begin{array}{r}
C r_{H}^{6} \gamma\left\{2 C r_{H}^{4}-q^{2}\left[2 C(2 C-1) \gamma^{2}+1\right]\right\} \phi_{H}^{\prime 2}+r_{H}\left\{2 C q^{4} \gamma^{2}+q^{2} r_{H}^{4}\left[4 C(C-1) \gamma^{2}+1\right]-2 C r_{H}^{8}\right\} \phi_{H}^{\prime} \\
+\gamma\left[12 C^{2} r_{H}^{8}-2(6 C-1) q^{2} r_{H}^{4}+q^{4}\right]=0
\end{array}
$$

The discriminant for $\gamma=\frac{1}{2}$ is

$$
\frac{1}{4} r_{H}^{2}\left(q^{2}-2 C r_{H}^{4}\right)^{2}\left[C^{2} q^{4}+2 C\left(6 C^{2}-3 C+4\right) q^{2} r_{H}^{4}+4\left(1-6 C^{2}\right) r_{H}^{8}\right]
$$

Our procedure for obtaining solutions is as follows. First we choose the suitable values of parameter $q$ and the boundary condition $\phi_{H}$ ( say $\phi_{H}=0$ ) at the horizon. Given the horizon radius $r_{H}$ together with $\phi_{H}^{\prime}$ determined by (5.1), we integrate the field equations (2.6) - (2.8) outward from the horizon numerically. There are two possible solutions for $\phi_{H}^{\prime}$ in Eq. (5.1), but only the smaller one gives the black hole solutions. Although the dilaton field takes nonvanishing value $\phi_{\infty}$ in the asymptotic region generically, it can be set to zero by using the shift symmetry (2.25). Note that the variables defined by

$$
x=e^{\gamma \phi_{H} / 2} r_{H}, \quad y=e^{(D-2) \gamma \phi_{H} / 2} q
$$

do not change under this shift symmetry. Then we redo this procedure by changing $r_{H}$ but keeping the values of charge $q$ and $\phi_{H}$ unchanged, which means that $y$ is also unchanged, and obtain another solution with the different asymptotic value of the dilaton field $\phi_{\infty}$. Using the shift symmetry to set $\phi_{\infty}$ to zero, we have the solution with the same $y$ but different $x$. In this way we obtain various solutions for one chosen $y$. Next, we repeat this procedure for different $y$ (practically, with different $q$ and the same $\phi_{H}$ ). After this process, we not only obtain solutions but also identify the parameter ranges where the solutions exist on the $x-y$ plane.

Since the values of $x$ and $y$ are unchanged by the shift symmetry (2.25), it is convenient to draw various diagrams in terms of these variables. $x$ and $y$ will be referred to as the "scaled horizon radius" and the "scaled charge", respectively. After identifying the parameter region where solutions exist, we can change these "scaled variables" to physical ones.

We find that there are black hole solutions in the region shadowed by horizontal thin (blue) lines in Fig. 1(a). The horizontal and vertical axes are $x$ and $y$, respectively. These horizontal (blue) lines actually consist of the sequences of solutions obtained by the above procedure. Although the lines are drawn for separate distance in $y$ with intervals $\Delta y=0.2$, it should be understood that there are solutions in the whole shadowed region. We call this region (where the black hole solutions exist) the allowed region. On the dashed (red) curve F determined by

$$
\mathrm{F}: y^{2}=\left[-2 x^{4}+3 x^{2}-12+\sqrt{3\left(x^{8}-4 x^{6}+27 x^{4}-24 x^{2}+48\right)}\right] x^{2},
$$




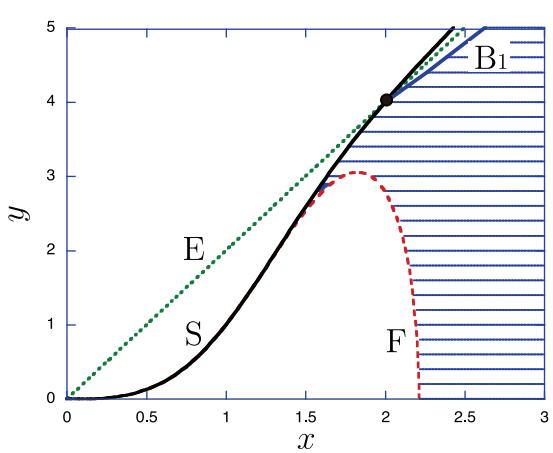

(a)

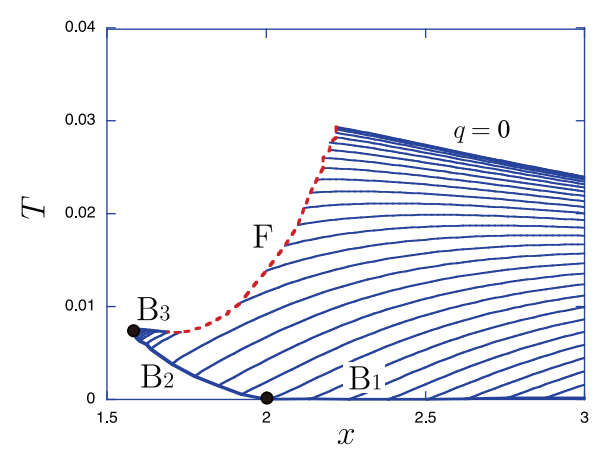

(c)

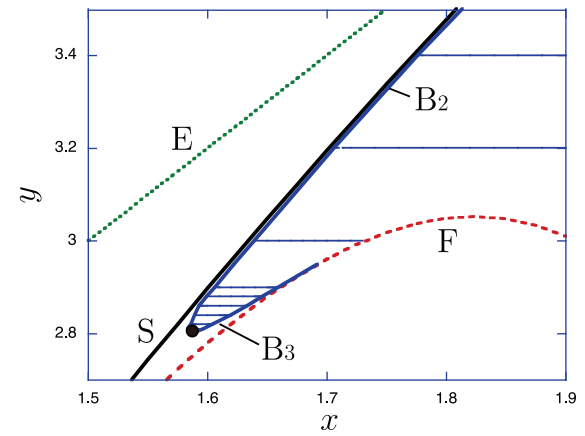

(b)

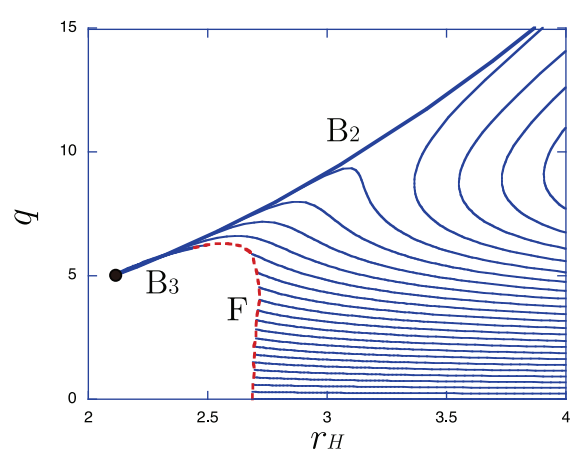

(d)

FIG. 1: The parameter region where the black hole solution exists in $D=4$. The black hole solution exists in the allowed region which is shadowed by (blue) thin lines. (a) The horizontal axis is $x=e^{\gamma \phi_{H} / 2} r_{H}$ and the vertical axis is $y=e^{\gamma \phi_{H}} q$. On the dashed (red) curve F, the discriminant (5.2) is zero. Below this line, the discriminant is negative and there is no appropriate boundary condition on the event horizon. On the curve $\mathrm{S}$ the first derivative of the dilaton field on the horizon $\phi_{H}^{\prime}$ diverges and the solution becomes singular. The line E represents the extreme solution. On the curve $\mathrm{B}_{1}, \phi_{\infty}$ diverges numerically which means $\phi_{H}$ diverges physically by the shift symmetry. (b) The magnified diagram around the top of the forbidden region. Around the curve $\mathrm{B}_{2}$ numerical calculation becomes unstable and we cannot find the black hole solution above it. On the curve $\mathrm{B}_{3}$, the third derivative of the dilaton field diverges while the Kretschmann invariant does not. (c) The allowed region in $x$ and the physical temperature $T$ of the black hole. The temperature becomes zero on the line $\mathrm{B}_{1}$. The reason is that the horizon of the solutions on it have infinite size. The solutions on the boundaries $\mathrm{F}, \mathrm{B}_{2}$, and $\mathrm{B}_{3}$ have non-zero finite temperature. (d) The diagram of the allowed region in terms of the physical quantity $r_{H}$ and $q$. For each value of the charge $q$, there is a lower bound for the horizon radius $r_{H}$.

the discriminant (5.2) vanishes. Below the curve F, the discriminant is negative and the values of $\phi_{H}^{\prime}$ becomes imaginary, and hence there is no black hole solution. Furthermore, on the curve F, it can be found analytically that the second derivative of the dilaton field diverges at the horizon. The Kretschmann invariant

$$
\begin{aligned}
\mathcal{K} & =R^{\mu \nu \rho \sigma} R_{\mu \nu \rho \sigma} \\
& =\left[B^{\prime \prime}-3 B^{\prime} \delta^{\prime}+2 B\left(\delta^{\prime 2}-\delta^{\prime \prime}\right)\right]^{2}+\frac{2(D-2)}{r^{2}}\left(B^{\prime 2}-2 B B^{\prime} \delta^{\prime}+2 B^{2} \delta^{\prime 2}\right)+\frac{2(D-2)_{3}}{r^{4}}(k-B)^{2},
\end{aligned}
$$

also diverges there, and the solution is singular. We call the region on and below the curve $\mathrm{F}$ the forbidden region. For $q=0$, the boundary is $x=24^{1 / 4} \approx 2.213$. For $y>3.0523$ (above the top of the curve $\mathrm{F}$ ), the discriminant is positive for all $x$ (and $r_{H}$ ).

The extremal condition given by $T_{H}=0$ (or $B_{H}^{\prime}=0$ ) is written in general dimensions as

$$
\mathrm{E}: y^{2}=2\left[(D-2)_{3} x^{2(D-3)}+(D-2)_{5} x^{2(D-4)}\right] .
$$

In four dimensions, this reduces to

$$
\mathrm{E}: y=2 x,
$$


and is depicted by the dotted (green) line $\mathrm{E}$. Above the line $\mathrm{E}, B_{H}^{\prime}$ is negative and the horizon $r_{H}$ is not a black hole horizon (i.e., a closed trapped surface). Actually we find that there is no black hole solution beyond the solid (black) curve $\mathrm{S}$, on which $\phi_{H}^{\prime}$ diverges. Hence the curve $\mathrm{S}$ is expected to give a boundary of the allowed region $($ for $y<4$ ). The equation of S can be read off from Eq. (5.1), as the condition that the coefficient of $\phi_{H}^{\prime 2}$ vanishes:

$$
\mathrm{S}: \quad y=2 x^{3} \sqrt{\frac{1}{x^{4}-x^{2}+4}} .
$$

For small $x(\lesssim 1)$, this singular curve $\mathrm{S}$ almost overlaps with the curve $\mathrm{F}$. And it crosses with the extreme curve $\mathrm{E}$ at $(x, y)=(2,4)$.

For $y \geq 4$, we find that the black hole solutions exist to the right of the boundary $\mathrm{B}_{1}$. On $\mathrm{B}_{1}$, we find that the asymptotic value of the dilaton field $\phi_{\infty}$ diverges in the numerical integration, which means that $\phi_{H}$ diverges after $\phi_{H}$ is shifted such that $\phi_{\infty} \rightarrow 0$ by the shift symmetry. Figure 1(b) is a magnified diagram around the top of forbidden region. For $3.0523<y<4$, we find that there seems to be a boundary $\mathrm{B}_{2}$ slightly to the right of the curve $\mathrm{S}$. We have confirmed that the black hole solutions exist on the right side of $\mathrm{B}_{2}$. In our numerical analysis, the calculation becomes unstable and stops for parameters just out of the event horizon before we let the parameters reach the curve $\mathrm{S}$, which strongly suggests that there exists the boundary $\mathrm{B}_{2}$. On the other hand, expanding the field equations around $r_{H}$, we do not find any singular behavior in the right region of $\mathrm{S}$ including $\mathrm{B}_{2}$ analytically. Unfortunately within our numerical accuracy, it is difficult to determine precisely where the true boundary is. However, there is certainly the boundary $\mathrm{B}_{2}$ different from $\mathrm{S}$ around $y \sim 2.8$, and it is plausible that it merges with $\mathrm{S}$ at the point $(x, y)=(2,4)$.

For $y<3.0523$, the allowed region splits into two parts. In the region to the right of the curve $\mathrm{F}$, the black hole solutions exist. On the left side of the curve F, we also have what we call solitary solutions. We find that the right boundary of the region where the solitary solutions exist is partly $\mathrm{F}$ and partly $\mathrm{B}_{3}$ (see Fig. 1(b)), where the third derivative of the dilaton field diverges at certain radius $r>r_{H}$ while the Kretschmann invariant $\mathcal{K}$ does not. The endpoint of $\mathrm{B}_{2}$ and $\mathrm{B}_{3}$ is depicted by a (black) dot. Below this, there are black hole solutions only to the right of $\mathrm{F}$.

In Fig. 1(c), we show the temperature vs. the scaled horizon radius relation. There are solutions in the region where (blue) thin curves are plotted. It should be noted that the temperature vanishes on the boundary $\mathrm{B}_{1}$, although $\mathrm{B}_{1}$ is different from the extreme curve $\mathrm{E}$. The reason why the temperature vanishes is that the asymptotic value of the dilaton field $\phi_{\infty}$ gets infinite and the horizon radius $r_{H}$ and the charge $q$ of the black hole on $\mathrm{B}_{1}$ become infinitely large after using the shift symmetry. The physical value of $\phi_{H}$ diverges and the solutions on $\mathrm{B}_{1}$ is singular. On the left lower boundary $\mathrm{B}_{2}$ around $x=1.6 \sim 2$ the temperature is non-zero. The right-upper boundary is determined by $q=0$, beyond which there is no solution.

The diagrams drawn in terms of $x$ and $y$ are useful to identify the parameter region numerically where the black hole solutions exist. To discuss the physical properties, however, it is more convenient to show the diagrams in terms of the physical quantities such as $r_{H}$ and $q$ directly. In Fig. 1(d), we display the allowed region in terms of the charge $q$ and the horizon radius $r_{H}$. There is a lower bound on the horizon radius for any value of charge $q$. When the charge is small $(q \lesssim 5)$, the curve F gives the boundary. We can see that the minimum size of the black hole is almost independent of the charge in this range. When the charge is in the range $5 \lesssim q \lesssim 6.3$, the curve $\mathrm{F}$ is again the boundary, but it is not the lower bound; there are solitary solutions in the thin region in the left part of the diagram. This cusp structure is surrounded by $\mathrm{B}_{2}$ and $\mathrm{B}_{3}$. The structure is so thin that it is difficult to create the black hole in this region in physical processes. For $q \gtrsim 6.3$, the lower boundary is given by $\mathrm{B}_{2}$. The curve $\mathrm{B}_{1}$ with $y \geq 2$ in Fig. 1(a) is pushed to the upper right infinitely $\left(r_{H} \rightarrow \infty, q \rightarrow \infty\right)$.

So far we have presented the discussion of the parameter region where black hole solutions exist. We now focus on how the field functions of the black hole solutions behave for a typical boundary condition. Figure 2 shows the behaviors of the function $\phi, m$ and $\delta$ for the horizon radius $r_{H}=3.2$ for neutral and charged cases with $q=0,5$ and 10. This horizon radius is chosen such that the solutions exist for these charges. For $q=10$, the parameters are close to the boundary $B_{2}$ in the diagram Fig. 1(d). We see that these functions have smooth behaviors. The dilaton field $\phi$ increases monotonically for each charge (Fig. 2(a)). The mass function $m$ of the neutral solution decreases near the horizon and increases towards a finite value as $r$ increases [16]. On the other hand the mass function of the charged solution increases all the range from the horizon (Fig. 2(b)). The lapse function $\delta$ decreases for small $q$. As the charge becomes large and the parameters approach $\mathrm{B}_{2}, \delta$ increases around the horizon and decreases to zero. Since the dilaton field couples to the gauge field and the GB term, the effects of the dilaton field and higher curvature terms become significant as the charge becomes large.

Up to this point, our discussions are rather qualitative with only figures of regions and behaviors of functions. To evaluate actually some quantities, it is necessary to have quantitative results. In order to get some idea on what are the typical physical quantities, here we tabulate them for the black hole solutions with the charge $q=0,5$ and 10 in Table I. 


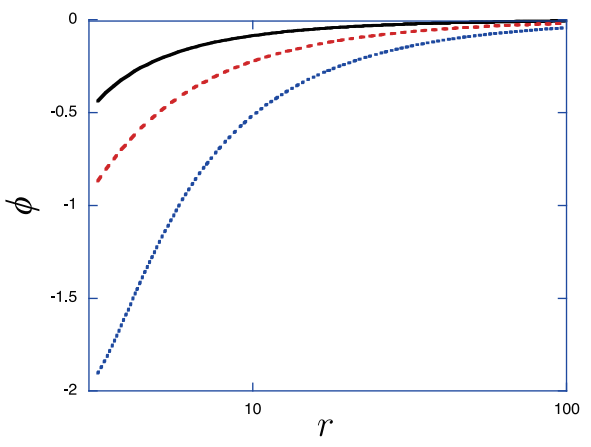

(a)

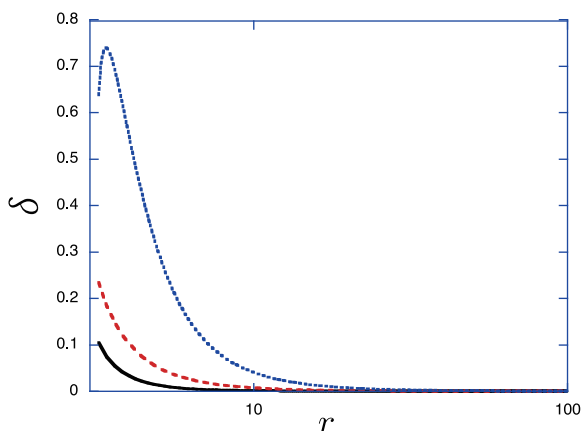

(c)

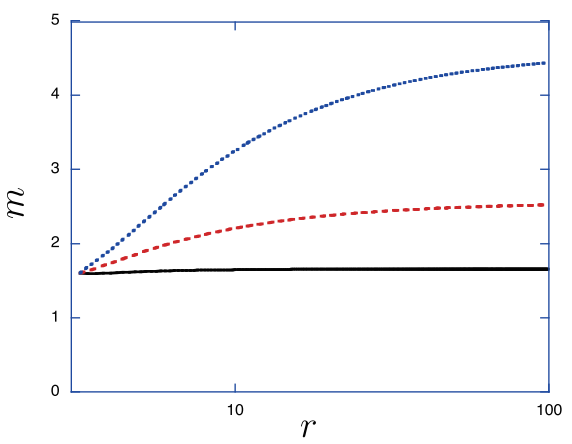

(b)

FIG. 2: Configurations of (a) the dilaton field $\phi$, (b) the mass function $m$, and (c) the lapse function $\delta$ of the black hole solutions in $D=4$. The horizon radii are $r_{H}=3.2$, and the charges are $q=0$ (solid (black) line), $q=5$ (dashed (red) line), $q=10$ (dotted (blue) line).

\begin{tabular}{c|c|c|c|c|c|c|c}
\hline$q$ & $r_{H}$ & $M$ & $\delta_{H}$ & $\phi_{H}$ & $\phi_{H}^{\prime}$ & $T$ & $S / \Sigma_{1}$ \\
\hline \hline \multirow{4}{*}{0} & 2.8 & 1.50205 & 0.291474 & -0.650704 & 0.504050 & 0.0282842 & 3.34452 \\
\cline { 2 - 8 } & 3.2 & 1.65494 & 0.104926 & -0.439547 & 0.253039 & 0.0248377 & 3.80579 \\
\cline { 2 - 8 } & 4.0 & 2.02371 & 0.0319963 & -0.256190 & 0.110000 & 0.0198896 & 5.13666 \\
\cline { 2 - 8 } & 6.0 & 3.00621 & 0.00521331 & -0.106409 & 0.0294481 & 0.0132626 & 10.0546 \\
\cline { 2 - 8 } & 8.0 & 4.00251 & 0.00156277 & -0.0586656 & 0.0120864 & 0.00994713 & 17.0298 \\
\hline \multirow{5}{*}{5} & 2.8 & 2.48068 & 0.502017 & -1.10819 & 0.564272 & 0.0143593 & 3.70037 \\
\cline { 2 - 8 } & 3.2 & 2.55368 & 0.234784 & -0.871222 & 0.347580 & 0.0143019 & 4.10591 \\
\cline { 2 - 8 } & 4.0 & 2.77132 & 0.0893922 & -0.582756 & 0.183430 & 0.0137251 & 5.33827 \\
\cline { 2 - 8 } & 6.0 & 3.51862 & 0.0168288 & -0.269283 & 0.0562110 & 0.0111825 & 10.1441 \\
\cline { 2 - 8 } & 8.0 & 4.38982 & 0.00522316 & -0.153149 & 0.0238626 & 0.00902933 & 17.0796 \\
\hline \multirow{2}{*}{10} & 2.8 & - & - & - & - & - & - \\
\cline { 2 - 8 } & 3.2 & 4.57779 & 0.635740 & -1.90468 & 0.285430 & 0.00099367 & 5.15176 \\
\cline { 2 - 8 } & 4.0 & 4.61313 & 0.529248 & -1.51849 & 0.413647 & 0.00404222 & 6.13667 \\
\cline { 2 - 8 } & 6.0 & 4.92428 & 0.112153 & -0.774269 & 0.154977 & 0.00651324 & 10.4728 \\
\cline { 2 - 8 } & 8.0 & 5.49493 & 0.0339071 & -0.445769 & 0.0650895 & 0.00667781 & 17.2497 \\
\hline
\end{tabular}

TABLE I: Typical values of the physical quantities of the black hole solutions in $D=4$.

Collecting all these information, we give summary of our results in the form of the relations between physical quantities of the black holes. Figure 3 shows various relations for neutral case (solid (black) line), charged case with $q=5$ (dashed (red) line) and with $q=10$ (dotted (blue) line). For comparison, we also give our results by the thick (thin) lines for dilatonic (non-dilatonic) solutions [see Eqs. (4.1)-(4.3) for the non-dilatonic case]. For the non-dilatonic solutions, the GB term becomes total divergence in $D=4$, and the solutions are Schwarzschild and RN black hole 


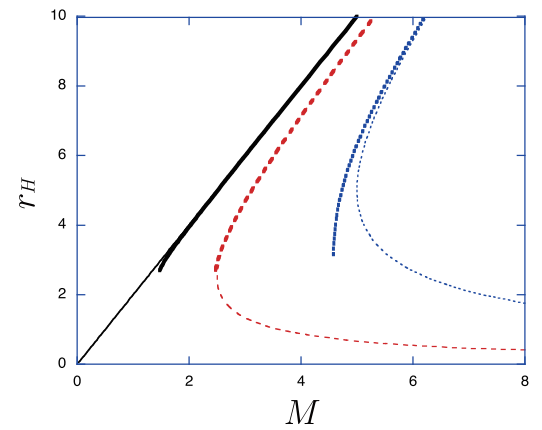

(a)

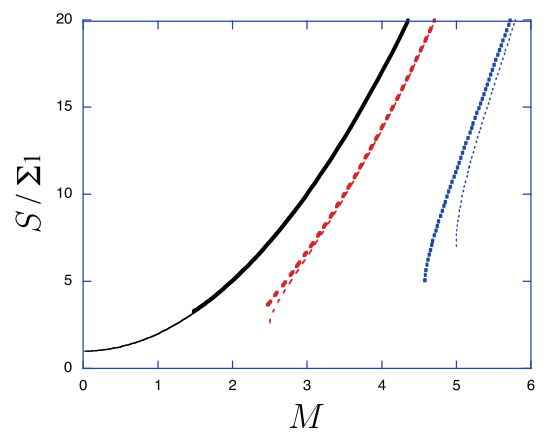

(c)

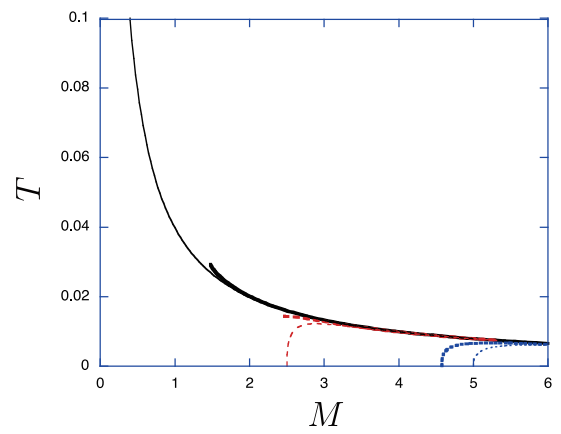

(b)

FIG. 3: Various relations for $D=4$ black hole solutions in (dilatonic) EGB systems. (a) $M-r_{H}$ diagram, (b) $M$ - $T$ diagram, (c) $M-S / \Sigma_{1}$ diagram. Solid (black) lines for $q=0$, dashed (red) lines for $q=5$, and dotted (blue) lines for $q=10$. Dilatonic solutions are given by thick lines and non-dilatonic ones by thin lines. In $D=4$, the non-dilatonic solutions are Schwarzschild $(q=0)$ and $\mathrm{RN}$ black hole $(q \neq 0)$ solutions.

solutions $\left(\bar{B}=1-2 \bar{M} / r+q^{2} / 4 r^{2}\right){ }^{3}$ For the RN black hole, there is the minimum mass for each charge, which corresponds to the extreme black hole solution. There are two values of $r_{H}$ for the fixed charge $q(\neq 0)$ and mass (see Fig. 3(a)). The larger is the radius of the black hole horizon, the smaller is that of the inner horizon of the same solution. In contrast, in the dilatonic case, there are lower bounds on the horizon radii as well as the masses of the black hole solutions for given fixed charges $q$. They are given as $(q, x, y)=(0,2.2134,0),(5,2.0117,2.7593)$, $(10,1.9686,3.9144)$. These solutions are not extremal. The lower bounds on the solutions with $q=0$ and 5 are determined by the forbidden region in Fig. 1; the parameters of the corresponding solutions are just on the boundary F. The horizon radii of the smallest black holes are almost the same for this range of charge as we have pointed out already, while their masses depend on the charge. For $q=10$, the lower bound corresponds to the singular solution not on $\mathrm{F}$ but on $\mathrm{B}_{2}$. From these figures, we see that the bigger the charge is, the further the curves move away from the neutral one, and the effect of the dilaton field is bigger. This is expected because the bigger the charge is, the bigger the dilaton coupling is.

Figures 3 (b) and 3 (c) give relations between the mass and temperature and their entropy, respectively. For the neutral case, the GB term has the tendency to raise the temperature compared to the non-dilatonic solution. For the charged case, however, the temperature is lower than the non-dilatonic solutions. The non-dilatonic solutions have extremal limit with $T=0$ while all the dilatonic solutions have non-zero finite temperature. This fact gives the following scenario. A dilatonic black hole loses its mass by emitting radiation and continues evaporating until the solution reaches the minimum mass solution and the spacetime becomes singular. The entropy of the Schwarzschild and RN black holes in the EGB theory is different from those in GR because of the second term in the square bracket of Eq. (3.3). This term is proportional to $\alpha^{\prime}$ and is absent in GR. In $D=4$, the contribution from this term to $S / \Sigma_{1}$ is 1 (constant). Hence, for instance, entropy of the Schwarzschild black hole in the zero mass limit is $S / \Sigma_{1}=1$ in the EGB theory.

\footnotetext{
3 The normalization of our charge is a factor 2 different from the conventional one.
} 


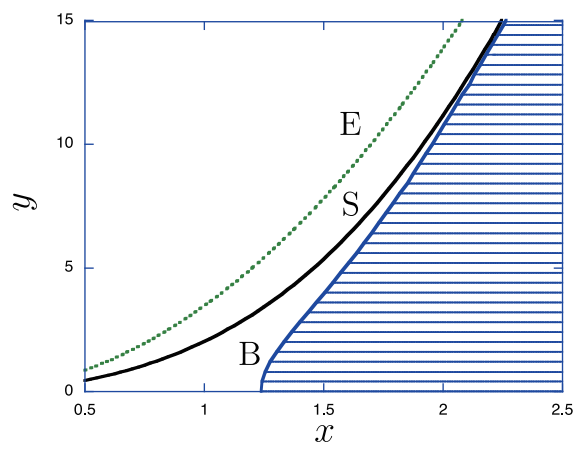

(a)

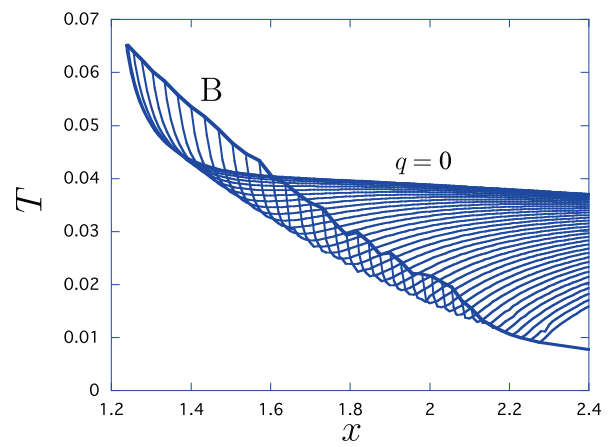

(b)

FIG. 4: The parameter region where the black hole solution exists in $D=5$. The black hole solution exists in the allowed region which is shadowed by (blue) thin lines. (a) The horizontal axis is $x=e^{\gamma \phi_{H} / 2} r_{H}$ and the vertical axis is $y=e^{3 \gamma \phi_{H} / 2} q$. On the curve $\mathrm{S}$ the first derivative of the dilaton field on the horizon $\phi_{H}^{\prime}$ diverges and the solution becomes singular. The curve E represents the extreme solution. The curve B gives the boundary where the solutions exist. On the curve B the second derivative of the dilaton field diverges at $r>r_{H}$ for $y \lesssim 12$. (b) The allowed region in terms of $x$ and the physical temperature $T$ of the black hole. The solutions on the boundary B have non-zero finite temperature.

\section{VI. $D=5$ BLACK HOLE}

For $D=5$, Eq. (2.18) to determine $\phi_{H}^{\prime}$ reduces to

$$
\begin{aligned}
C r_{H}^{8} & \gamma\left\{q^{2}\left[3 C(4 C-1) \gamma^{2}+C+1\right]-3 C r_{H}^{6}\left(3 C^{2} \gamma^{2}+C+1\right)\right\} \phi_{H}^{\prime 2} \\
& +r_{H}\left\{(C+1)^{2} r_{H}^{6}\left(3 C r_{H}^{6}-q^{2}\right)-2 C \gamma^{2}\left[9 C^{2}(C+1) r_{H}^{12}+3(C-1)(2 C+1) q^{2} r_{H}^{6}+2 q^{4}\right]\right\} \phi_{H}^{\prime} \\
& +\gamma\left[9 C^{2}\left(3 C^{2}+2 C-3\right) r_{H}^{12}+3\left(3 C^{2}+6 C-1\right) q^{2} r_{H}^{6}-2 q^{4}\right]=0 .
\end{aligned}
$$

The discriminant is

$$
\begin{aligned}
\frac{1}{2} r_{H}^{2}\left(q^{2}-3 C r_{H}^{6}\right)^{2}\left[18 C^{6} r_{H}^{12}+30 C^{5} r_{H}^{12}+C^{4} r_{H}^{6}\left(12 q^{2}+5 r_{H}^{6}\right)+2 C^{3} r_{H}^{6}\left(11 q^{2}-8 r_{H}^{6}\right)\right. \\
\left.+C^{2}\left(2 q^{4}+3 q^{2} r_{H}^{6}-12 r_{H}^{12}\right)+8 C r_{H}^{6}\left(q^{2}+r_{H}^{6}\right)+2 r_{H}^{12}\right] .
\end{aligned}
$$

We show the allowed region where the black hole solutions exist in Fig. 4(a). Since the discriminant (6.2) is always positive for $C>0$, there is no forbidden region. This is also the case for higher dimensions. There are, however, other bounds given by the curve $\mathrm{S}$ on which the first derivative of the dilaton field diverges at the horizon. There is also the curve $\mathrm{E}$, on which the solution becomes extreme. The equations of these curves are

$$
\begin{gathered}
\mathrm{S}: \quad y=2 x^{2} \sqrt{\frac{3\left(x^{4}+4 x^{2}+12\right)}{x^{4}+x^{2}+48}}, \\
\mathrm{E}: \quad y=2 \sqrt{3} x^{2}
\end{gathered}
$$

respectively. The actual boundary of the allowed region is given by the curve B. The interval of each thin line in the allowed region is $\Delta y=0.4$. The thin lines in other figures in this section have the same interval.

For $y \lesssim 12, \phi_{H}$ is large near the boundary B so that the physical horizon radius $r_{H}$ and the charge $q$ are very small as we will see soon (recall that our physical $\phi_{H}$ is determined by the shift symmetry (2.25)). This implies that the solution has the similar properties to the neutral solution. (The neural solution with higher order term of the dilaton field $(a=1)$ is summarized in the Appendix.) The neutral solution also has the lower bound on $r_{H}$, where the second derivative of the dilaton field diverges at outer region $r>r_{H}$. We find that the same divergence occur also in the charged case. Kretschmann invariant diverges there. Hence the outer domain of the black hole with parameters on B $(y \lesssim 12)$ is singular. For larger $y(\gtrsim 12.4), \phi^{\prime \prime}$ diverges at radius close to the horizon $r \approx r_{H}$ for solutions with parameters on B. For larger $y$, the extreme curve $\mathrm{E}$ and $\mathrm{S}$ intersect at $(x, y)=(2 \sqrt{3}, 24 \sqrt{3})=(3.464,41.57)$ but the boundary B is still below the extreme curve E. 


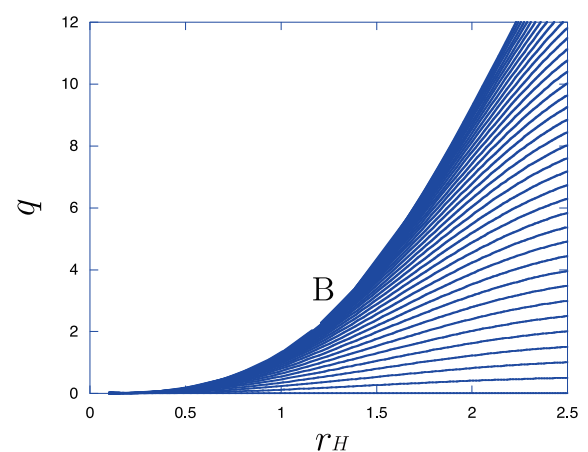

(a)

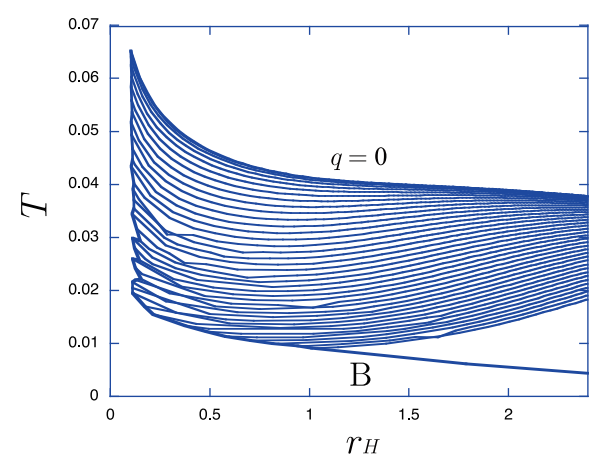

(b)

FIG. 5: The parameter region where the black hole solutions exist in $D=5$. (a) The diagram of the horizon radius $r_{H}$ and the charge $q$. For each value of the charge $q$, there is a lower bound for the horizon radius $r_{H}$. (b) The diagram of the horizon radius $r_{H}$ and the temperature $T$.

In Fig. 4(b), we show the allowed region in terms of the scaled horizon radius $x$ and the temperature $T$. Each thin curve has the same $y$. The allowed region is three-dimensional with another axis of $y$, and the boundary looks to turn around into inside the region. On the boundary represented by $\mathrm{B}$, the temperature of the minimum size solution is finite, hence a small black hole may evolve to this solution through the evaporating process. For $y \lesssim 12$ there may appear the singularity at non-zero radius away from the horizon. On the other hand, for $y \gtrsim 12.4$, the singularity appears at the radius very close to the horizon just before the radiation stops. The qualitative difference of the boundary B with $y \lesssim 12$ or $y \gtrsim 12.4$ mentioned above appears around $x=2.1$ in the diagram. The boundary curve B decreases rapidly there, and no "fold" is observed for $x \gtrsim 2.1$.

We also give the allowed region in terms of $r_{H}, q$ and $T$ in Fig. 5. The curve B corresponds to the boundary in Fig. 4. In Fig. 5(a) we see that the solutions with the same $y$ (depicted by thin lines) converge to the lower left corner corresponding to neutral solution. For example, the physical parameter of the solution with $(x, y)=(2.02,11)$ is $\left(r_{H}, q\right)=(0.177,0.0848)$. The boundary B changes its qualitative property at $\left(r_{H}, q\right) \sim(0.2,0.1)(y \approx 12)$. Figure $5(\mathrm{~b})$ shows that the temperature gets lower in the presence of the charge but it does not vanish for all solutions. The left vertical boundary of B corresponds to $y \lesssim 12$.

Figure 6 shows the configurations of the field functions $\phi, m$ and $\delta$ for the horizon radius $r_{H}=2.1$ for neutral and charged cases with $q=0,5$ and 10 . This horizon radius is chosen such that the solutions exist for these charges. There are some different features in $D=5$ dimension compared to the $D=4$ case; the dilaton field decreases and the lapse function increases rapidly around the event horizon. Although the configurations in the neutral case are qualitatively different depending on the horizon radius (details can be found in Ref. [16]), we find that the variations of the field functions become large as the charge becomes large in general due to the dilaton coupling as in the $D=4$ case.

Here we tabulate physical quantities for the black hole solutions with the charge $q=0,5$ and 10 in Table II.

In Fig. 7, we give the relations of physical quantities for neutral and charged solutions with different charges as well as those in non-dilatonic theory. For the neutral case, the non-dilatonic black hole has the zero horizon radius limit where $\bar{M}=1$ (Fig. $7(\mathrm{a})$ ). It is a singular solution. There is also a lower bound on the horizon radius for the dilatonic solution. For the minimum solution, the second derivative of the dilaton field diverges outside the horizon. For the charged case, the non-dilatonic solution is described by Eqs. (4.1) and (4.3). There is the extreme solution for each charge, which has the minimum mass. As in the $\mathrm{RN}$ black holes in $D=4$, the lower curve for each charge is the radius of the inner horizon. The graphs of the charged dilatonic solution show similar behavior of the neutral one except for the radius of the lowest mass solution. For the minimum solution, the second derivative of the dilaton field diverges just outside the horizon.

We can see from Fig. 7(b) that the non-dilatonic solutions has zero temperature in the low mass limit regardless of the charge. For the charge case, the dilatonic solution behaves like non-dilatonic one. However, the temperature of the neutral solution raises as the mass becomes small. Figure $7(\mathrm{c})$ shows the mass-entropy relations. 


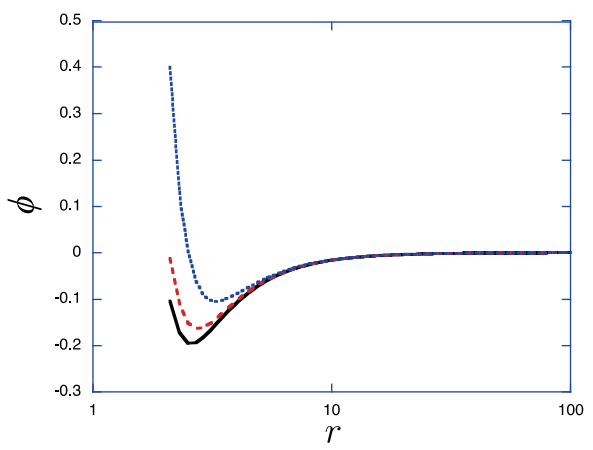

(a)

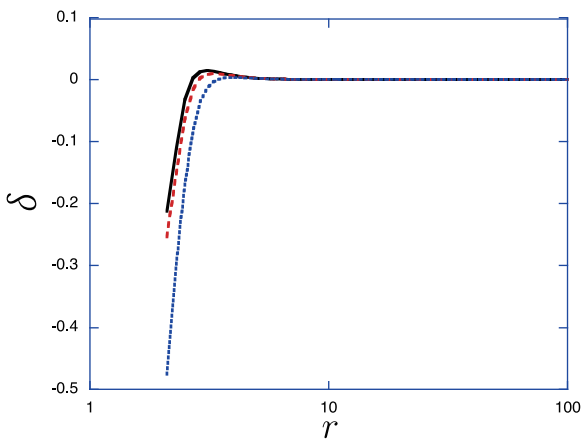

(c)

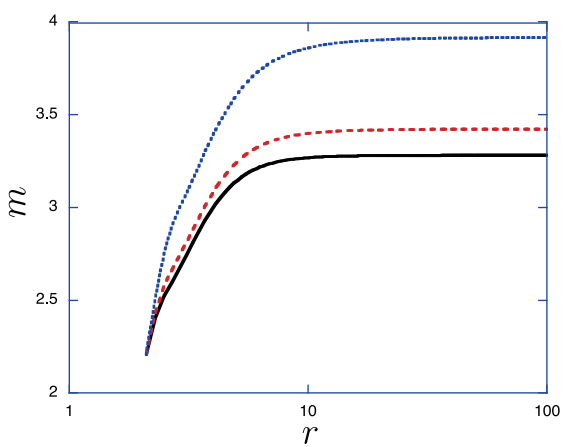

(b)

FIG. 6: Configurations of (a) the dilaton field $\phi$, (b) the mass function $m$, and (c) the lapse function $\delta$ of the black hole solutions in $D=5$. The horizon radii are $r_{H}=2.1$, and the charges are $q=0$ (solid (black) line), $q=5$ (dashed (red) line), $q=10$ (dotted (blue) line).

\begin{tabular}{|c|c|c|c|c|c|c|c|}
\hline$q$ & $r_{H}$ & $M$ & $\delta_{H}$ & $\phi_{H}$ & $\phi_{H}^{\prime}$ & $T$ & $S / \Sigma_{1}$ \\
\hline \multirow[t]{5}{*}{0} & 0.2 & 0.25377 & 0.512040 & 7.37067 & -19.7397 & 0.0563593 & 0.0170506 \\
\hline & 1.7 & 2.21038 & -0.341768 & 0.268320 & -1.08980 & 0.0395517 & 5.68795 \\
\hline & 2.2 & 3.56697 & -0.173250 & -0.168216 & -0.346471 & 0.0383783 & 9.84113 \\
\hline & 4.0 & 9.43189 & 0.065321 & -0.305025 & 0.172855 & 0.0313079 & 29.9771 \\
\hline & 6.0 & 19.2458 & 0.0181629 & -0.164383 & 0.0680353 & 0.0237666 & 73.5419 \\
\hline \multirow[t]{5}{*}{5} & 0.2 & - & - & - & - & - & - \\
\hline & 1.7 & 2.41969 & -0.484009 & 0.678086 & -2.25502 & 0.0254686 & 4.86175 \\
\hline & 2.2 & 3.69719 & -0.208188 & -0.0987342 & -0.464995 & 0.0352647 & 9.59600 \\
\hline & 4.0 & 9.49343 & 0.064932 & -0.306446 & 0.171797 & 0.0310818 & 29.9870 \\
\hline & 6.0 & 19.2748 & 0.0182388 & -0.165188 & 0.0682174 & 0.0237302 & 73.5498 \\
\hline \multirow[t]{5}{*}{10} & 0.2 & - & - & - & - & - & - \\
\hline & 1.7 & - & - & - & - & - & - \\
\hline & 2.2 & 4.14235 & -0.343864 & 0.157902 & -1.05281 & 0.0236971 & 8.76096 \\
\hline & 4.0 & 9.68000 & 0.0637826 & -0.310739 & 0.168659 & 0.0304058 & 30.0171 \\
\hline & 6.0 & 19.3627 & 0.0184687 & -0.167607 & 0.068766 & 0.0236212 & 73.5735 \\
\hline
\end{tabular}

TABLE II: Typical values of the physical quantities of the black hole solutions in $D=5$. 


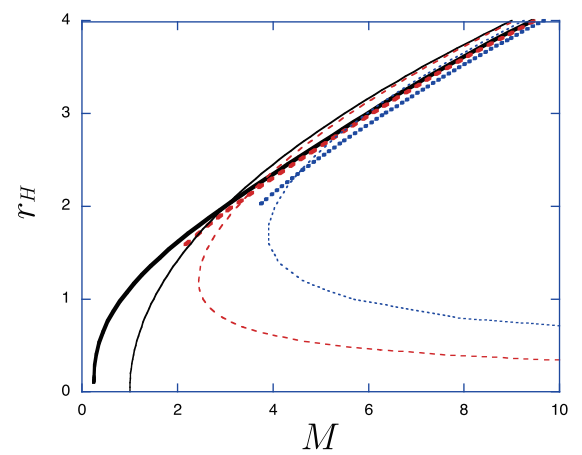

(a)

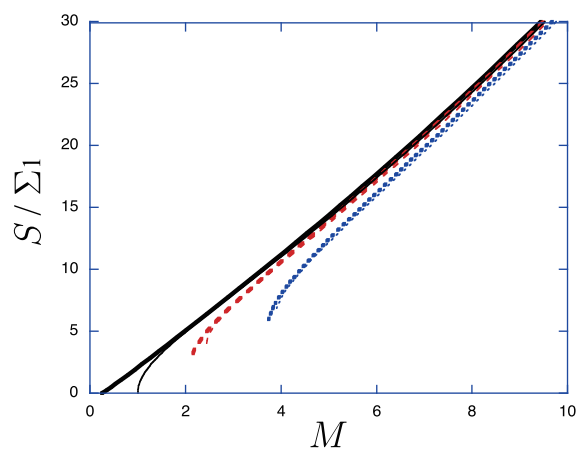

(c)

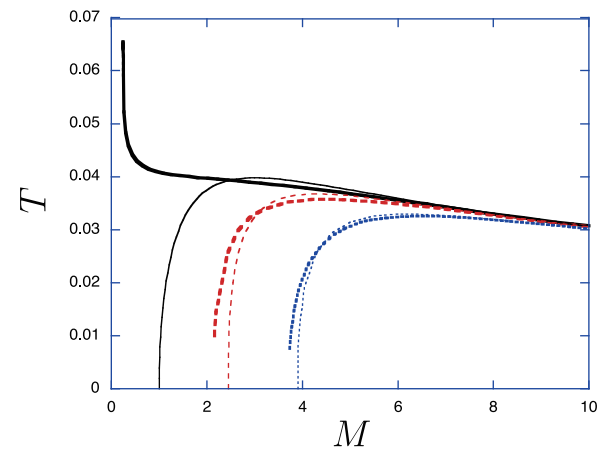

(b)

FIG. 7: Various relations for $D=5$ black hole solutions in (dilatonic) EGB systems. (a) $M-r_{H}$ diagram, (b) $M-T$ diagram, (c) $M-S / \Sigma_{1}$ diagram. Solid (black) lines for $q=0$, dashed (red) lines for $q=5$, and dotted (blue) lines for $q=10$. Dilatonic solutions are given by thick lines and non-dilatonic ones by thin lines.

\section{VII. $D=6$ BLACK HOLE}

For $D=6$, Eq. (2.18) to determine $\phi_{H}^{\prime}$ reduces to

$$
\begin{aligned}
& C r_{H}^{10} \gamma\left\{4 C r_{H}^{8}\left[4 C^{2}(6 C+7) \gamma^{2}+C(2 C+7)+3\right]-3 q^{2}\left[4 C(6 C-1) \gamma^{2}+2 C+1\right]\right\} \phi_{H}^{2} \\
& \quad-r_{H}\left\{2 C \gamma^{2}\left[80 C^{2}\left(C^{2}-C-1\right) r_{H}^{16}-12(C-1)(4 C+1) q^{2} r_{H}^{8}-9 q^{4}\right]+(2 C+1)^{2} r_{H}^{8}\left[4 C(C+3) r_{H}^{8}-3 q^{2}\right]\right] \phi_{H}^{\prime} \\
& \quad-\gamma\left[80 C^{2}\left(7 C^{2}+2 C-2\right) r_{H}^{16}+12\left(6 C^{2}+6 C-1\right) q^{2} r_{H}^{8}-9 q^{4}\right]=0
\end{aligned}
$$

The discriminant is

$$
\begin{aligned}
& \frac{1}{4} r_{H}^{2} {\left[3 q^{2}-4 C(C+3) r_{H}^{8}\right]^{2}\left[400 C^{6} r_{H}^{16}+480 C^{5} r_{H}^{16}+24 C^{4} r_{H}^{8}\left(5 q^{2}+6 r_{H}^{8}\right)+8 C^{3} r_{H}^{8}\left(15 q^{2}-4 r_{H}^{8}\right)\right.} \\
&\left.+C^{2}\left(9 q^{4}+36 q^{2} r_{H}^{8}+16 r_{H}^{16}\right)+8 C r_{H}^{8}\left(3 q^{2}+4 r_{H}^{8}\right)+4 r_{H}^{16}\right]
\end{aligned}
$$

which is always positive for $C>0$. Hence again there is no forbidden region from the existence of the proper boundary condition in the $x$ - $y$ diagram in Fig. 8 . There is, however, the region where solutions cease to exist if we keep $y$ constant and make $x$ smaller. The curve $\mathrm{S}$ where $\phi_{H}^{\prime}$ diverges and the curve $\mathrm{E}$ where solution becomes extreme are expressed as

$$
\begin{gathered}
\mathrm{S}: y=2 x^{2} \sqrt{\frac{6\left(x^{6}+14 x^{4}+108 x^{2}+432\right)}{x^{4}+6 x^{2}+216}}, \\
\mathrm{E}: \quad y=2 x^{2} \sqrt{6\left(x^{2}+2\right)},
\end{gathered}
$$

respectively. The curves $\mathrm{S}$ and $\mathrm{E}$ intersect at $(x, y)=(2 \sqrt{5}, 80 \sqrt{33})$. 


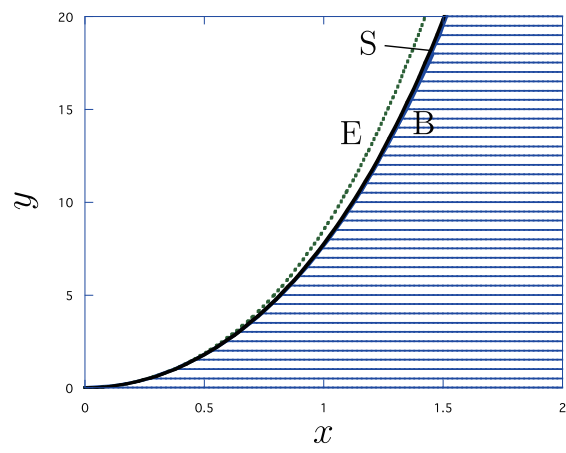

(a)

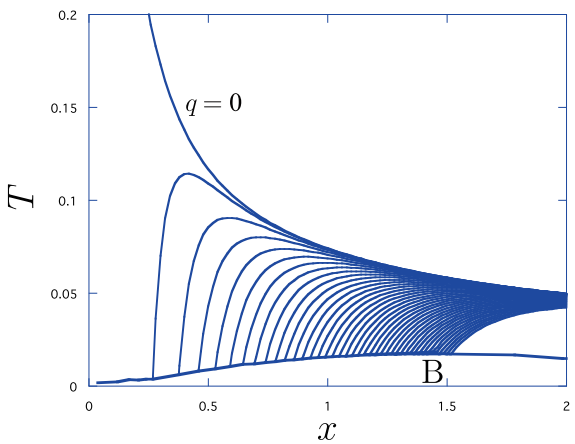

(c)

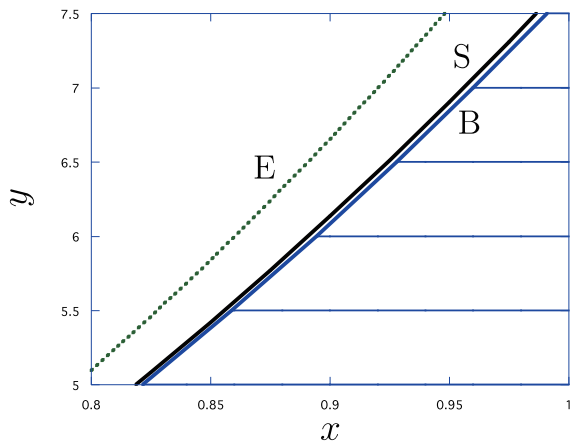

(b)

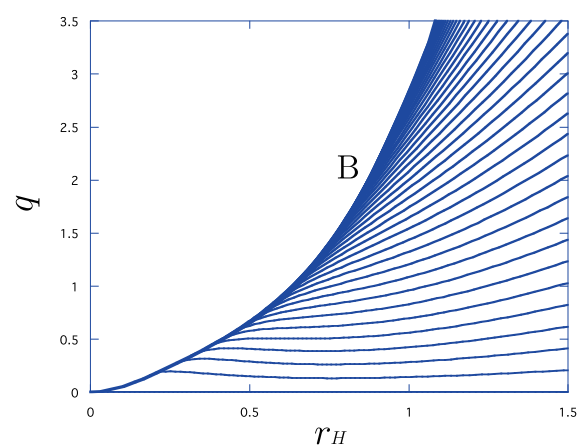

(d)

FIG. 8: The parameter region where the black hole solution exists in $D=6$. The black hole solution exists in the allowed region which is shadowed by (blue) thin lines. (a) The horizontal axis is $x=e^{\gamma \phi_{H} / 2} r_{H}$ and the vertical axis is $y=e^{3 \gamma \phi_{H} / 2} q$. On the curve $\mathrm{S}$ the first derivative of the dilaton field on the horizon $\phi_{H}^{\prime}$ diverges and the solution becomes singular. The line E represents the extreme solution. The curve B gives the boundary where the solutions exist. On the boundary B the second derivative of the dilaton field diverges just outside the horizon. (b) The magnified diagram of (a). The boundary B is separated from the singular curve S. (c) The allowed region in $x$ and the physical temperature $T$ of the black hole. There are solutions above the curve B on the right side. The solutions on the boundaries B have non-zero finite temperature. The boundary B seems to go down to the origin. (d) The diagram of the allowed region in terms of the physical quantity $r_{H}$ and $q$. For each value of the charge $q$, there is a lower bound for the horizon radius $r_{H}$.

For the neutral case, there is a solution in the zero horizon limit, while there is the lower bound for $x$ for the charged solution as shown in Fig. 8(a). We find that the boundary is given by B where $\phi^{\prime \prime}$ diverges just outside the horizon $\left(r \approx r_{H}\right)$. Kretschmann invariant also diverges there. In $D=5$, the second derivative of the dilaton field diverges at $r>r_{H}$ on the boundary, but there is not such behavior in $D \geq 6$. The interval of the thin lines in the allowed region is $\Delta y=0.5$. Figure $8(\mathrm{~b})$ is the magnified diagram to distinguish $\mathrm{B}$ from $\mathrm{S}$. The difference between them is small. Hence the boundary B is well approximated by Eq. (7.3) for $x \lesssim 2 \sqrt{5}$. From Fig. 8(c), the temperature does not vanish on the boundary B but it is lower than that of closer solutions on the same curve. On the boundary B, the temperature decreases as $y \rightarrow 0$, which corresponds to zero charge limit, because the boundary B approaches to the extreme curve $\mathrm{E}$ in this limit. However, for the neutral solution with $y=0$ exactly, the temperature diverges in $x \rightarrow 0$ limit. This disconnected behavior is the same as that between the Schwarzschild and the RN black hole solutions in GR. There are solutions above the curve B on the right side (although we do not fill in curves). The boundary B seems to go down to the origin. In Fig. 8(d), we display the allowed region in terms of physical quantities $r_{H}$ and $q$. The left boundary in Fig. 8(d) is B, to the left of which there is no solution.

Figure 9 shows the configurations of the field functions $\phi, m$ and $\delta$ for the horizon radius $r_{H}=1.6$ for neutral and charged cases with $q=0,5$ and 10 .

We tabulate quantitative results on the physical quantities for the black hole solutions with the charge $q=0,5$ and 10 in Table III.

Figure 10 show the relation between physical quantities for some fixed charges. Figure 10(a) shows the mass and horizon radius relation. The neutral black holes have the zero mass and zero horizon radius limit for both the dilatonic and non-dilatonic cases. As in the lower dimensional cases the radius of the inner horizon of the non-dilatonic solution is depicted. In $D=6$, the horizon radius of the dilatonic solution is larger than that of the non-dilatonic solution for the same mass, and the difference between them is remarkable. On the other hand, the dependence of the charge 


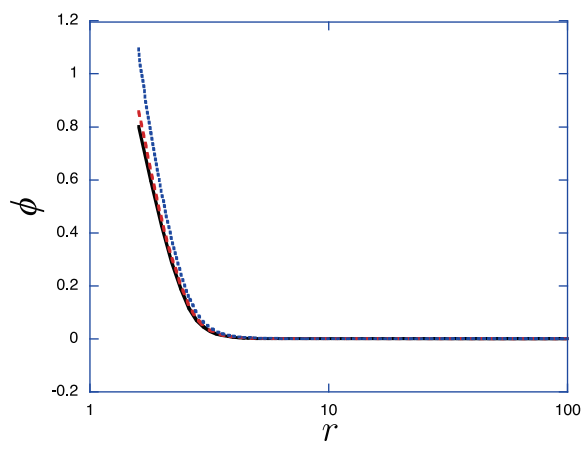

(a)

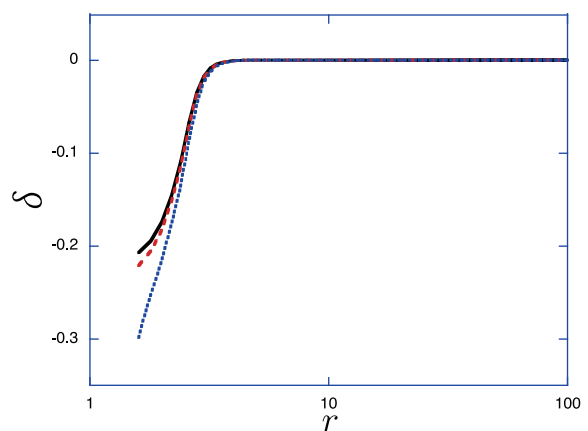

(c)

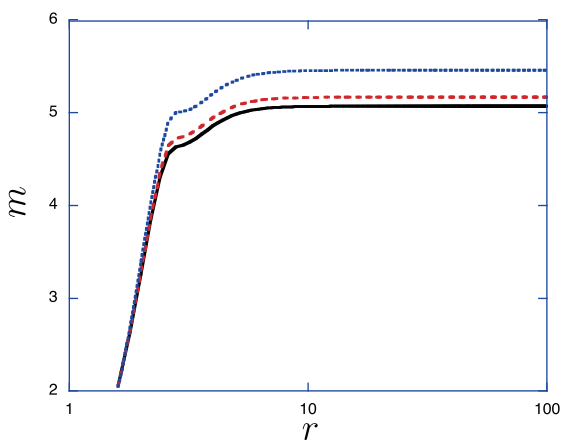

(b)

FIG. 9: Configurations of the functions; (a) the dilaton field $\phi$, (b) the mass function $m$ and (c) the lapse function $\delta$ of the black hole solutions in $D=6$. The horizon radii are $r_{H}=1.6$, and the charges are $q=0$ (solid (black) line), $q=5$ (dashed (red) line), $q=10$ (dotted (blue) line).

\begin{tabular}{|c|c|c|c|c|c|c|c|}
\hline$q$ & $r_{H}$ & $M$ & $\delta_{H}$ & $\phi_{H}$ & $\phi_{H}^{\prime}$ & $T$ & $S / \Sigma_{1}$ \\
\hline \multirow[t]{5}{*}{0} & 0.2 & 0.468100 & -0.0468327 & 0.687743 & -0.325345 & 0.209961 & 0.170565 \\
\hline & 1.7 & 5.76616 & -0.205696 & 0.725035 & -1.05676 & 0.0492691 & 14.1553 \\
\hline & 2.2 & 10.3889 & -0.182805 & 0.385590 & -0.713747 & 0.0454031 & 29.8043 \\
\hline & 4.0 & 45.6455 & -0.00965932 & -0.157190 & 0.00409228 & 0.0378379 & 167.850 \\
\hline & 6.0 & 129.375 & 0.0230420 & -0.177248 & 0.0797759 & 0.0312294 & 560.017 \\
\hline \multirow[t]{5}{*}{5} & 0.2 & - & - & - & - & - & - \\
\hline & 1.7 & 5.84751 & -0.215021 & 0.760954 & -1.12794 & 0.0472207 & 13.9405 \\
\hline & 2.2 & 10.4274 & -0.184416 & 0.391221 & -0.723068 & 0.0450363 & 29.7369 \\
\hline & 4.0 & 45.6528 & -0.00969279 & -0.157154 & 0.00400513 & 0.0378301 & 167.848 \\
\hline & 6.0 & 129.377 & 0.0230421 & -0.177253 & 0.0797760 & 0.0312288 & 560.017 \\
\hline \multirow[t]{5}{*}{10} & 0.2 & - & - & - & - & - & - \\
\hline & 1.7 & 6.09473 & -0.253558 & 0.892202 & -1.43403 & 0.0402291 & 13.1877 \\
\hline & 2.2 & 10.5425 & -0.189437 & 0.408529 & -0.752338 & 0.0439171 & 29.5312 \\
\hline & 4.0 & 45.6745 & -0.00979316 & -0.157046 & 0.00374374 & 0.0378066 & 167.842 \\
\hline & 6.0 & 129.393 & 0.0230423 & -0.177268 & 0.0797761 & 0.0312269 & 560.019 \\
\hline
\end{tabular}

TABLE III: Typical values of the physical quantities of the black hole solutions in $D=6$.

in the dilatonic case is rather mild. We can see that the $r_{H}-M$ graph of each charge traces almost the same curve. The left end points of the curves correspond to singular solutions with the parameters on B. Figure 10(b) shows the relation between the mass $M$ and the temperature $T$. There appear some qualitative differences depending on whether the solution is charged or not especially around the endpoint in the small mass limit. Figure 10(c) shows the relation between entropy and the mass. Compared with the $r_{H}-M$ diagram, the graphs of dilatonic solutions are separated from each other. Here it should be noted that the entropy is not proportional to the horizon area in the 


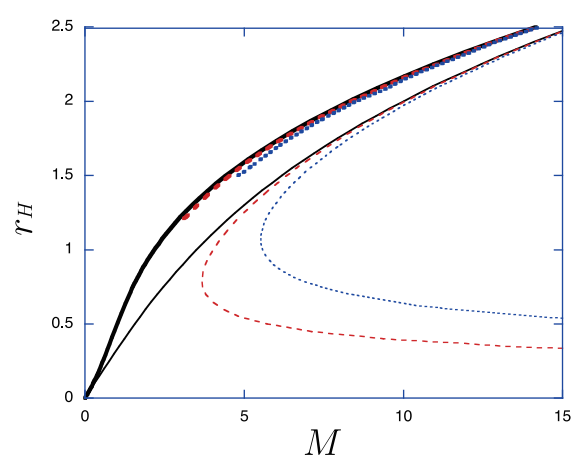

(a)

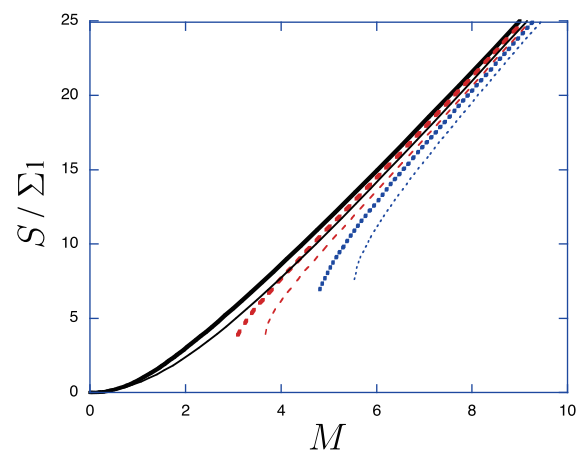

(c)

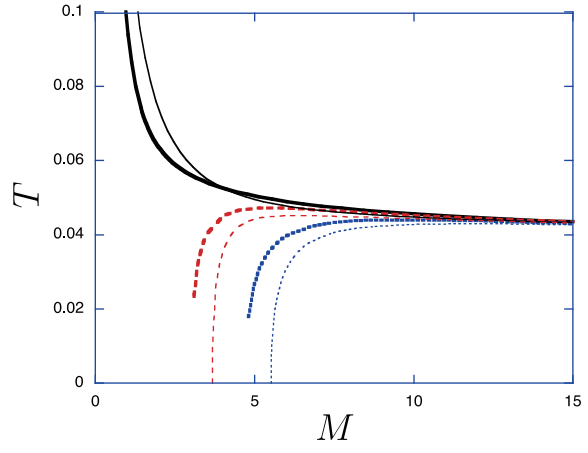

(b)

FIG. 10: The relations of physical quantities for black hole solutions in $D=6$ EGB systems. (a) $M-r_{H}$ diagram, (b) $M-T$ diagram, (c) $M-S / \Sigma_{1}$ diagram. Solid (black) line for $q=0$, dashed (red) line for $q=5$, and dotted (blue) line for $q=10$. The dilatonic solutions are given by thick lines, and the non-dilatonic ones by thin lines.

higher curvature theory. The expression of entropy is given by Eq. (3.3), and the second term in the square bracket gives this separation.

\section{VIII. $D=10$ BLACK HOLE}

The qualitative properties in the $D=6$ to 10 cases are almost the same. However, since $D=10$ is the critical dimension in superstring theories and important for applications, here we show the summary and some diagrams of the black hole solutions in $D=10$.

For $D=10$, Eq. (2.18) to determine $\phi_{H}^{\prime}$ reduces to

$$
\begin{aligned}
C r_{H}^{18} \gamma\left\{8 C r_{H}^{16}\left[24 C^{2}(70 C+19) \gamma^{2}+90 C^{2}+57 C+7\right]-7 q^{2}\left[8 C(14 C-1) \gamma^{2}+6 C+1\right]\right\} \phi_{H}^{\prime 2} \\
-r_{H}\left\{8 C r_{H}^{32}\left[432 C^{2}\left(15 C^{2}-C-1\right) \gamma^{2}+540 C^{3}+108 C^{2}+99 C+7\right]\right. \\
\left.-\quad 7 q^{2} r_{H}^{16}\left[16 C(C-1)(12 C+1) \gamma^{2}+(6 C+1)^{2}\right]-98 C q^{4} \gamma^{2}\right\} \phi_{H}^{\prime} \\
-\gamma\left[576 C^{2}\left(99 C^{2}+6 C-4\right) r_{H}^{32}+56\left(18 C^{2}+6 C-1\right) q^{2} r_{H}^{16}-49 q^{4}\right]=0 .
\end{aligned}
$$

The discriminant is

$$
\begin{aligned}
\frac{1}{4} r_{H}^{2} & {\left[7 q^{2}-8 C(15 C+7) r_{H}^{16}\right]^{2}\left[46656 C^{6} r_{H}^{32}+34560 C^{5} r_{H}^{32}+432 C^{4}\left(7 q^{2} r_{H}^{16}+20 r_{H}^{32}\right)\right.} \\
+ & \left.192 C^{3}\left(7 q^{2} r_{H}^{16}+9 r_{H}^{32}\right)+C^{2}\left(7 q^{2}+24 r_{H}^{16}\right)^{2}+8 C\left(7 q^{2} r_{H}^{16}+12 r_{H}^{32}\right)+4 r_{H}^{32}\right]
\end{aligned}
$$

which is always positive. Hence again there is no forbidden region in the parameter space from this restriction. 


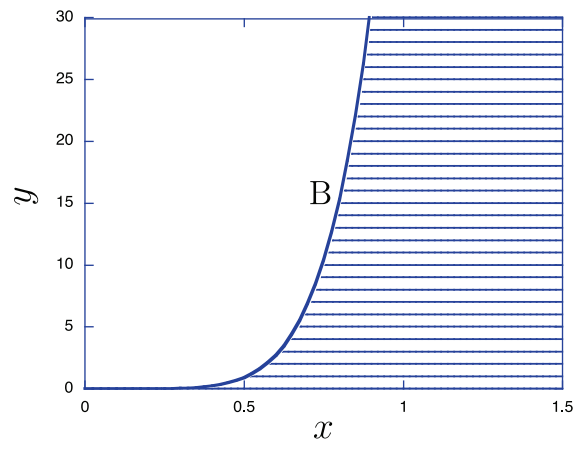

(a)

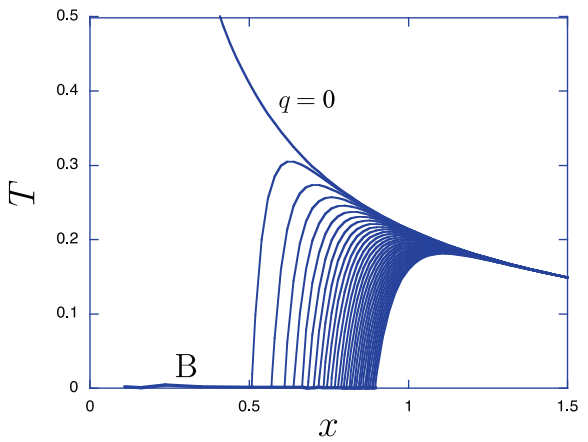

(c)

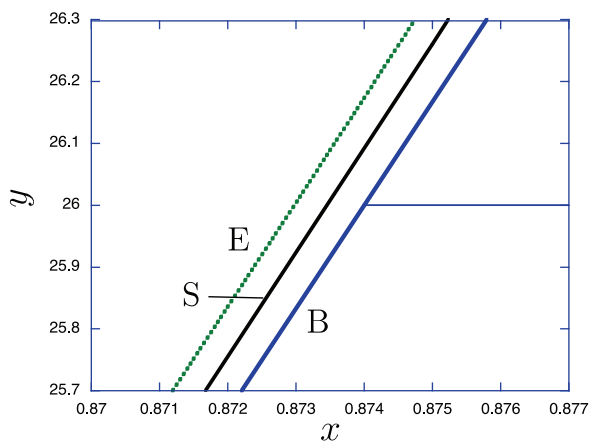

(b)

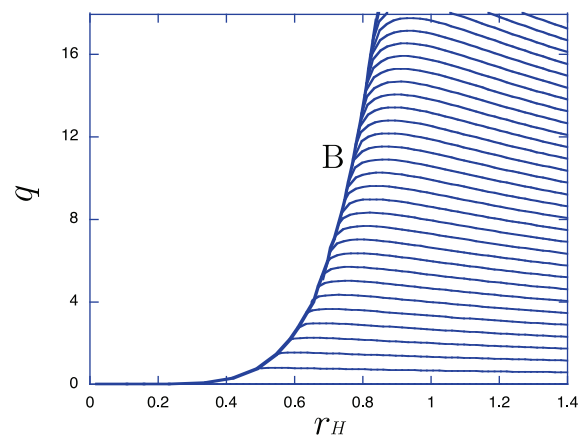

(d)

FIG. 11: The parameter region where the black hole solution exists in $D=10$. The black hole solution exists in the allowed region which is shadowed by (blue) thin lines. (a) The horizontal axis is $x=e^{\gamma \phi_{H} / 2} r_{H}$ and the vertical axis is $y=e^{4 \gamma \phi_{H}} q$. The curve B gives the boundary where the solutions exist. On the boundary B the second derivative of the dilaton field diverges just outside the horizon. (b) The magnified diagram of (a). There are small differences between the boundary B, the singular curve S, and the extreme curve E. (c) Allowed region in $x$ and the physical temperature $T$ of the black hole. There are solutions also in the lower-right region. The solutions on the boundaries B have non-zero finite temperature. The boundary B seems to go down to the origin. (d) The diagram of the allowed region in terms of the physical quantity $r_{H}$ and $q$. For each value of the charge $q$, there is a lower bound for the horizon radius $r_{H}$.

In Fig. 11, we give the allowed region for the dilatonic black holes. The interval of the thin lines in the allowed region is $\Delta y=1$. In Fig. 11(a), the extremal curve $\mathrm{E}$ and the singular curve $\mathrm{S}$ where $\phi_{H}^{\prime}$ diverges overlap with the boundary $\mathrm{B}$, which are expressed as

$$
\begin{gathered}
\text { S: } y=4 x^{6} \sqrt{\frac{7\left(x^{6}+114 x^{4}+5712 x^{2}+164640\right)}{x^{4}+56 x^{2}+5488}}, \\
\text { E: } y=4 x^{6} \sqrt{7\left(x^{2}+30\right)},
\end{gathered}
$$

respectively. The curves $\mathrm{S}$ and $\mathrm{E}$ intersect at $(x, y)=(2 \sqrt{13}, 162432 \sqrt{574})$. Figure 11(b) is the magnified diagram of (a). We can see that there are actually small differences between these curves. On the boundary B, the second derivative of the dilaton field diverges just outside the horizon. Figure 11(c) shows the $T-x$ diagram. There are solutions between the $q=0$ curve and the boundary B. On the boundary B the temperature is not zero. In Fig. 11(d), we display the allowed region in terms of $x$ and $y$. Because the value of $\phi_{H}(<0.3)$ is small for all ranges, there is not much difference from Fig. 11(a).

The configurations of the field functions $\phi, m$ and $\delta$ are qualitatively similar to $D=6$ case, and we omit the figures here. Instead, we give quantitative results on the typical physical quantities for the black hole solutions with the charge $q=0,5$ and 10 in Table IV.

In Fig. 12, we give the relations of physical quantities. There is not much difference between the dilatonic and the non-dilatonic solutions for these values of charge. This seems to be the tendency for higher-dimensional solutions. 


\begin{tabular}{c|c|c|c|c|c|c|c}
\hline$q$ & $r_{H}$ & $M$ & $\delta_{H}$ & $\phi_{H}$ & $\phi_{H}^{\prime}$ & $T$ & $S / \Sigma_{1}$ \\
\hline \hline \multirow{6}{*}{0} & 0.2 & 0.0066645 & -0.00168778 & 0.0355222 & -0.0319461 & 0.996737 & 0.00176109 \\
\cline { 2 - 8 } & 1.7 & 6.48277 & -0.0125900 & 0.163543 & -0.133113 & 0.253318 & 6.80565 \\
\cline { 2 - 8 } & 2.2 & 276.002 & -0.0368148 & 0.318372 & -0.276744 & 0.125172 & 593.831 \\
\cline { 2 - 8 } & 4.0 & 1027.74 & -0.0497577 & 0.361146 & -0.333154 & 0.100354 & 2787.35 \\
\cline { 2 - 8 } & 6.0 & 26445.5 & -0.0693803 & 0.285279 & -0.335072 & 0.0646700 & 115826 \\
\hline \multirow{5}{*}{5} & 0.2 & - & - & - & - & - & - \\
\cline { 2 - 8 } & 1.7 & 7.06843 & -0.0138779 & 0.170767 & -0.147951 & 0.221395 & 6.80611 \\
\cline { 2 - 8 } & 2.2 & 276.008 & -0.0368154 & 0.318375 & -0.276748 & 0.125170 & 593.831 \\
\cline { 2 - 8 } & 4.0 & 1027.74 & -0.0497577 & 0.361147 & -0.333155 & 0.100354 & 2787.35 \\
\cline { 2 - 8 } & 6.0 & 26445.5 & -0.0693803 & 0.285279 & -0.335072 & 0.0646700 & 115826 \\
\hline \multirow{4}{*}{10} & 0.2 & - & - & - & - & - & - \\
\cline { 2 - 8 } & 1.7 & 8.76069 & -0.0207554 & 0.193860 & -0.236732 & 0.123396 & 6.70390 \\
\cline { 2 - 8 } & 2.2 & 276.018 & -0.0368170 & 0.318383 & -0.276759 & 0.125164 & 593.828 \\
\cline { 2 - 8 } & 4.0 & 1027.74 & -0.0497578 & 0.361147 & -0.333155 & 0.100354 & 2787.35 \\
\cline { 2 - 8 } & 6.0 & 26445.5 & -0.0693803 & 0.285279 & -0.335072 & 0.0646700 & 115826 \\
\hline
\end{tabular}

TABLE IV: Typical values of the physical quantities of the black hole solutions in $D=10$.

\section{CONCLUSIONS}

In this paper we have studied asymptotically flat charged black hole solutions in the dilatonic EGB theory in various dimensions. The theory is the low-energy effective theory of the heterotic string. The spacetime is assumed to be static and spherically symmetric. The system of the field equations is so complex that it is difficult to obtain an analytical solution. Hence we investigate the numerical solutions. The system of the field equations has some symmetries which are helpful in the analysis. The results are given for $D=4,5,6$ and 10, and we did not discuss other dimensions 7 to 9 simply because we expect that behaviors are qualitatively the same as those presented here from our earlier study of the system.

We have found that there is the forbidden region on the parameter plane spanned by $x$ and $y$ which are the "scaled horizon radius" and the "scaled charge", respectively, in $D=4$. Besides it, there are some boundaries (and important curves) of the allowed region such as the singular curve $\mathrm{S}$ where $\phi_{H}^{\prime}$ diverges, the extreme curve $\mathrm{E}$ where the solution becomes extreme, and $\mathrm{B}_{i}(i=1,2,3)$ where the field functions and/or its derivatives diverge. The forms of the allowed parameter regions are different depending on the dimension, 4, 5, and $6-10$.

We have also studied the thermodynamical quantities. There is no extreme black hole solution with $T=0$ that can be obtained by taking the limit of the non-extreme solutions within the parameter range we chose. In the higher curvature theory, physical entropy is defined by Iyer and Wald [32]. Although there is a parameter region where the radius (or the area) of the black hole horizon in the dilatonic theory is smaller than that in the non-dilatonic theory, entropy in the dilatonic theory is always larger than that in the non-dilatonic theory. Since the dilatonic and non-dilatonic solutions are the solutions in different theories, it is difficult to compare their thermodynamical stability and quantum transition between them in the thermodynamical sense as long as $\gamma \neq 0$. There would be, however, some physical meaning in this magnitude relationship of entropy.

It is noted again that our analysis includes the higher order term of the dilaton field which is not in our previous works [16-20]. To make its effects clear, we have studied the solutions for both cases $a=0$ and $a=1$ in Eq. (2.4) in the Appendix by focusing on the neutral solutions. The qualitative properties and the relations such as $M-r_{H}, M-T$ and $M-S / \Sigma_{1}$ are quite similar in each case except for $D=5$. In the $D=5$ theory without the higher order term, the black hole solution with infinitesimal size exists towards $r_{H}=0$ while there is a lower bound on the horizon radius $r_{H}$ in the theory with the higher order term. For the lower bound solution, the second derivative of the dilaton field diverges at some radius outside the horizon, and the spacetime becomes singular. The existence of the lower bound of this type is confirmed also in the charged solution with $y \lesssim 12(q \lesssim 0.1)$.

By our analysis it is found that the properties of the black hole solutions strongly depend on the dimension, charge, existence of the dilaton field. Hence both the detailed analyses of the individual systems and the investigations from the systematic point of view are important.

There still remain some questions in our work. The first one is to determine precisely where the real boundary of the allowed region $\mathrm{B}\left(\right.$ or $\left.\mathrm{B}_{2}\right)$ is. It is difficult to determine it due to the fine structure of the system near the boundary and the numerical accuracy. The second is concerned with the fact that the temperature of the black hole solutions on 


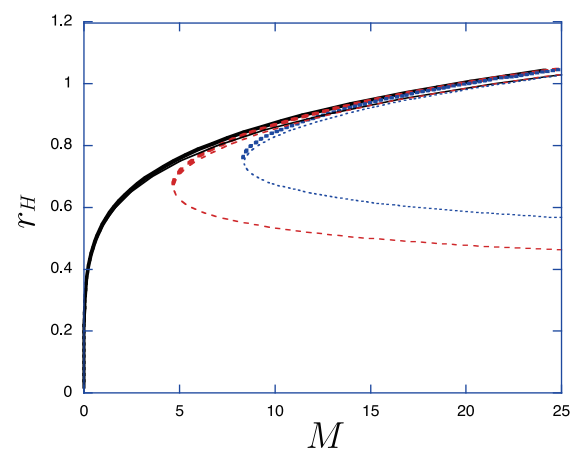

(a)

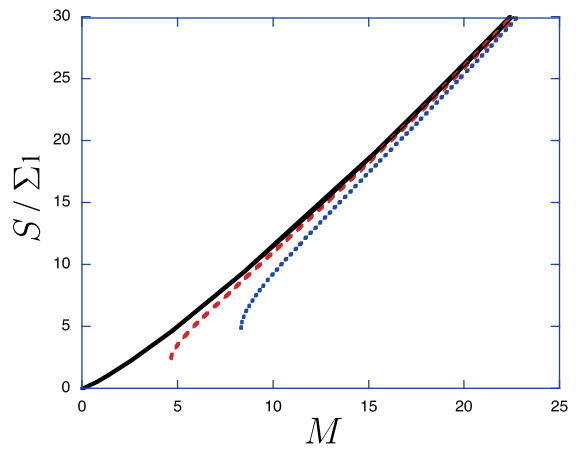

(c)

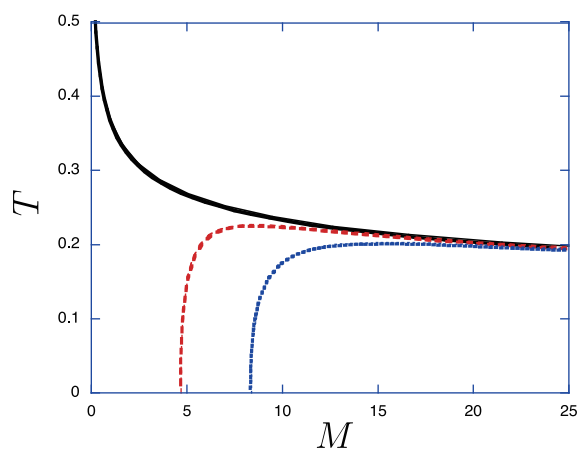

(b)

FIG. 12: The relations of various physical quantities for black hole solutions in $D=10$ dilatonic EGB systems. (a) $M-r_{H}$ diagram, (b) $M-T$ diagram, (c) $M-S / \Sigma_{1}$ diagram. Solid (black) line for $q=0$, dashed (red) line for $q=5$, and dotted (blue) line for $q=10$. The dilatonic solutions are given by thick lines, and the non-dilatonic ones by thin lines.

some boundary remains non-zero and finite. This means that the evaporation does not stop there and the black hole still evolves to a naked singularity or something unknown. This gives a very interesting puzzle that the singularity may be really formed after evaporation process and deserves further study.

Another interesting future work is to study charged AdS black holes for application to AdS/CFT correspondence. We expect that this class of solutions exists for $k=0$ [17]. We leave these problems for future works.

\section{Acknowledgements}

This work was supported in part by the Grant-in-Aid for Scientific Research Fund of the JSPS (C) Grant No. 24540290, (C) Grant No. 22540293and, and (A) Grant No. 22244030.

\section{Appendix A: The effects of the higher order term of the dilaton field for the neutral solutions}

In our previous work on the black holes in the dilatonic EGB theory [16-20], we examined the neutral solutions without the higher order derivative term of the dilaton field ( $a=0$ in Eq. (2.4)). There is, however, such a term in general. Hence it is significant to investigate if there are any differences between the cases of $a=0$ and $a=1$. The neutral solutions for $a=1$ can be obtained by putting $q=0$ in our model. One can find the result by looking at the curves with $q=0$ in Figs. 1-3 for $D=4$. Comparing them with the $a=0$ case in our previous papers, we find that the qualitative properties and the relations such as $M-r_{H}, M-T$ and $M-S / \Sigma_{1}$ are quite similar. This is true also in other dimensions except for five. Hence let us discuss $D=5$ case in more detail here.

Figure 13 displays the relations between physical quantities for neutral solutions. The black hole solutions in the theory with the GB term have the non-zero lower bound on their mass. Compared with the non-dilatonic BD solution, the mass of the lower bound in the dilatonic solutions is smaller, and the one for $a=1$ is slightly smaller than that for 


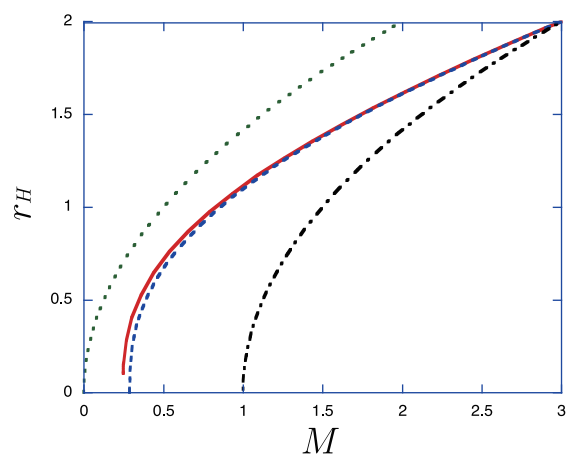

(a)

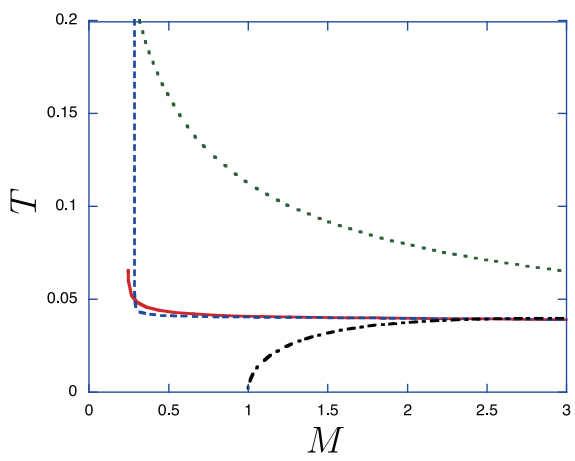

(c)

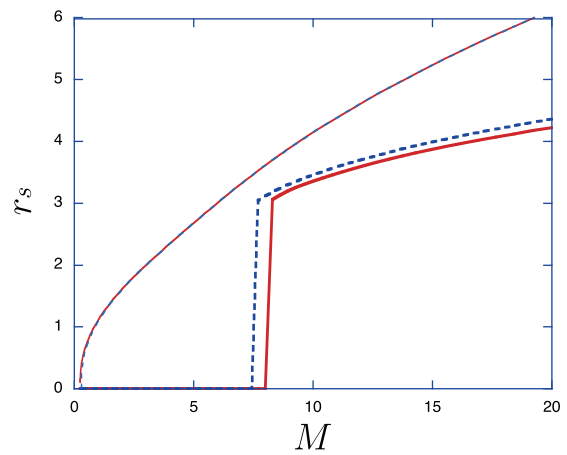

(b)

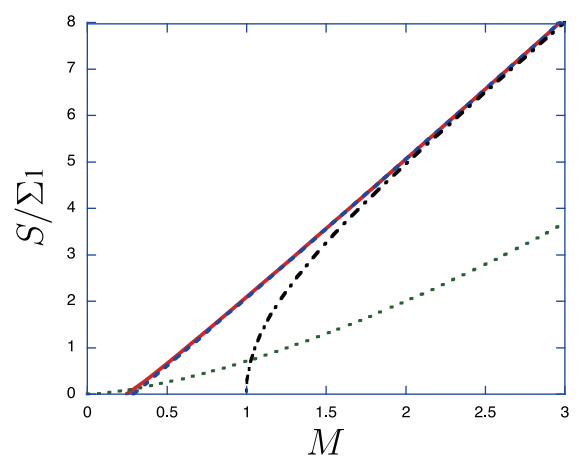

(d)

FIG. 13: Difference between $a=1$ and $a=0$ for neutral black hole solutions in the $D=5$ dilatonic EGB system with $\gamma=1 / 2$. (a) $M-r_{H}$ diagram, (b) $M-r_{s}$ diagram (The thin lines show the horizon radius), (c) $M-T$ diagram, (d) $M-S / \Sigma_{1}$ diagram. The solid (red) line is for $a=1$, the dashed (blue) line for $a=0$, the dotted (green) line for Tangherini solution (higher-dimensional generalization of the Schwarzschild solution without the GB term), and the dotted-dashed (black) line for the BD solution (in non-dilatonic EGB theory).

$a=0$ (Fig. 13(a)). We also find that for the $a=0$ case the black hole solution with infinitesimal size exists towards $r_{H}=0$ while there is a lower bound on the horizon radius $r_{H}$ for $a=1$. This bound corresponds to the boundary B in Figs. 4(a) and 5(a). With the parameters around the lower bound B the second derivative of the dilaton field grows suddenly at some radius $r>r_{H}$. This behavior is shown in Fig. 14 for the parameters just before touching the boundary. It is expected that $\phi^{\prime \prime}$ should diverge on the boundary. Since the Kretschmann invariant also diverges at that radius, the solution of the lower bound is singular. If we compare black holes with the same mass, the horizon radius for $a=1$ is a little larger than that for $a=0$. As a general tendency, values of the dilaton field $\phi$ itself and its variation $\phi^{\prime}$ are larger in magnitude for $a=1$ than those for $a=0$. For the large black holes $\left(r_{H} \gtrsim 1\right)$, the differences are indistinguishable.

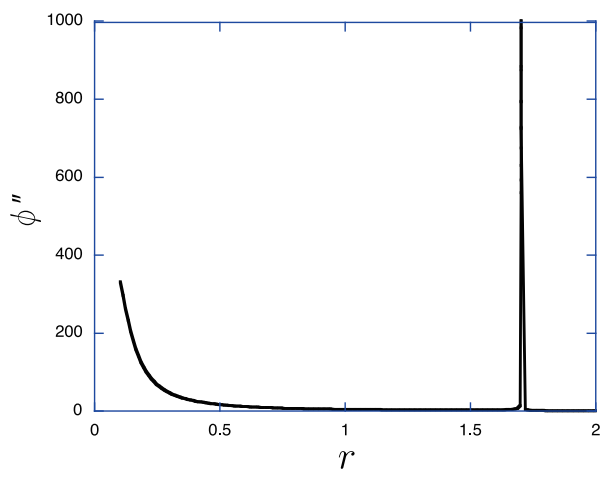

FIG. 14: Configuration of $\phi^{\prime \prime}$ for parameters $q=0, r_{H}=0.103555$ just before touching the lower bound in $D=5$. The peak is at $r=1.702$. This behavior is basically the same for the charged solutions with $y<12(q<0.1)$.

Figure 13(b) shows the radius of the curvature singularity (thick curves) inside the black hole horizon (of which 
radius is depicted by thin curves). For $M \lesssim 7.5$, the curvature singularity is located at the center of the black hole. For the large mass $M \gtrsim 7.5$, however, the singularity is expanded to a finite radius $r_{s}>0$. We called it a fat singularity [20]. Even if the higher order term of the dilaton field is included, this tendency is unchanged.

The temperature $T$ is slightly higher in $a=1$ than in $a=0$ case for $M \gtrsim 0.6$, while the order changes near the minimum mass (Fig. 13(c)). Although it might appear that the difference is very small in $D=5$, the difference is further smaller in other dimensions. In addition, the temperature of the minimum size solution is finite, hence a small black hole for $a=1$ may evolve to this solution through the evaporating process, and the naked singularity might appear at non-zero radius. Such behavior cannot be seen for higher dimensions even if the higher order term is included. Figure $13(\mathrm{~d})$ is the entropy vs. mass diagram.

[1] G. W. Gibbons and K. -i. Maeda, Nucl. Phys. B 298 (1988) 741.

[2] D. Garfinkle, G. T. Horowitz and A. Strominger, Phys. Rev. D 43 (1991) 3140 [Erratum-ibid. D 45 (1992) 3888].

[3] R. R. Metsaev and A. A. Tseytlin, Nucl. Phys. B 293 (1987) 385.

[4] D. Lovelock, J. Math. Phys. 12 (1971) 984; 13 (1972) 874.

[5] B. Zumino, Phys. Rep. 137 (1986) 109.

[6] J. T. Wheeler, Nucl. Phys. B 268 (1986) 737;

D. L. Wiltshire, Phys. Lett. B 169 (1986) 36;

R. C. Myers and J. Z. Simon, Phys. Rev. D 38 (1988) 2434;

G. Giribet, J. Oliva and R. Troncoso, JHEP 0605 (2006) 007 [arXiv:hep-th/0603177];

R. G. Cai and N. Ohta, Phys. Rev. D 74 (2006) 064001 [arXiv:hep-th/0604088].

For reviews and references, see C. Garraffo and G. Giribet, arXiv:0805.3575 [gr-qc] and C. Charmousis, arXiv:0805.0568 $[\mathrm{gr}-\mathrm{qc}]$.

[7] M. Cvetic, S. Nojiri and S. D. Odintsov, Nucl. Phys. B 628 (2002) 295 [arXiv:hep-th/0112045].

[8] P. Kanti, N. E. Mavromatos, J. Rizos, K. Tamvakis and E. Winstanley, Phys. Rev. D 54 (1996) 5049 [arXiv:hepth/9511071].

[9] T. Torii, H. Yajima and K. -i. Maeda, Phys. Rev. D 55 (1997) 739 [gr-qc/9606034].

[10] D. G. Boulware and S. Deser, Phys. Rev. Lett. 55 (1985) 2656; Phys. Lett. B 175 (1986) 409.

[11] J. D. Brown, J. Creighton and R. B. Mann, Phys. Rev. D 50 (1994) 6394 [gr-qc/9405007].

[12] R. G. Cai, Phys. Rev. D 65 (2002) 084014 [arXiv:hep-th/0109133].

[13] A. Buchel and J. T. Liu, Phys. Rev. Lett. 93 (2004) 090602 [arXiv:hep-th/0311175];

P. Kovtun, D. T. Son and A. O. Starinets, Phys. Rev. Lett. 94 (2005) 111601 [arXiv:hep-th/0405231];

A. Buchel, J. T. Liu and A. O. Starinets, Nucl. Phys. B 707 (2005) 56 [arXiv:hep-th/0406264];

M. Brigante, H. Liu, R. C. Myers, S. Shenker and S. Yaida, Phys. Rev. Lett. 100 (2008) 191601 [arXiv:0802.3318 [hep-th]].

[14] R. -G. Cai, Z. -Y. Nie, N. Ohta and Y. -W. Sun, Phys. Rev. D 79 (2009) 066004 [arXiv:0901.1421 [hep-th]].

[15] C. G. Callan, Jr., R. C. Myers and M. J. Perry, Nucl. Phys. B 311 (1989) 673.

[16] Z. K. Guo, N. Ohta and T. Torii, Prog. Theor. Phys. 120 (2008) 581 [arXiv:0806.2481 [gr-qc]].

[17] Z. K. Guo, N. Ohta and T. Torii, Prog. Theor. Phys. 121 (2009) 253 [arXiv:0811.3068 [gr-qc]].

[18] N. Ohta and T. Torii, Prog. Theor. Phys. 121 (2009) 959 [arXiv:0902.4072 [hep-th]].

[19] N. Ohta and T. Torii, Prog. Theor. Phys. 122 (2009) 1477 [arXiv:0908.3918 [hep-th]].

[20] N. Ohta and T. Torii, Prog. Theor. Phys. 124 (2010) 207 [arXiv:1004.2779 [hep-th]].

[21] K. Bamba, Z. K. Guo and N. Ohta, Prog. Theor. Phys. 118 (2007) 879 [arXiv:0707.4334 [hep-th]].

[22] T. Torii and H. Maeda, Phys. Rev. D 71 (2005) 124002 [arXiv:hep-th/0504127]; Phys. Rev. D 72 (2005) 064007 [arXiv:hepth/0504141].

[23] C. M. Chen, D. V. Gal'tsov and D. G. Orlov, Phys. Rev. D 75 (2007) 084030 [arXiv:hep-th/0701004].

[24] C. M. Chen, D. V. Gal'tsov and D. G. Orlov, Phys. Rev. D 78 (2008) 104013 [arXiv:0809.1720 [hep-th]].

[25] R. G. Cai, C. M. Chen, K. -i. Maeda, N. Ohta and D. W. Pang, Phys. Rev. D 77 (2008) 064030 [arXiv:0712.4212 [hep-th]].

[26] K. -i. Maeda, N. Ohta and Y. Sasagawa, Phys. Rev. D 80 (2009) 104032 [arXiv:0908.4151 [hep-th]];

[27] C. M. Chen, D. V. Gal'tsov, N. Ohta and D. G. Orlov, Phys. Rev. D 81 (2010) 024002 [arXiv:0910.3488 [hep-th]].

[28] K. -i. Maeda, N. Ohta and Y. Sasagawa, Phys. Rev. D 83 (2011) 044051 [arXiv:1012.0568 [hep-th]].

[29] C. Charmousis, B. Gouteraux and E. Kiritsis, JHEP 1209 (2012) 011 [arXiv:1206.1499 [hep-th]].

[30] S. S. Gubser, Phys. Rev. D 78 (2008) 065034 [arXiv:0801.2977 [hep-th]].

[31] K. -i. Maeda, N. Ohta and R. Wakebe, Eur. Phys. J. C 72 (2012) 1949 [arXiv:1111.3251 [hep-th]].

[32] R. M. Wald, Phys. Rev. D 48 (1993) 3427 [arXiv:gr-qc/9307038]; V. Iyer and R. M. Wald, Phys. Rev. D 50 (1994) 846 [arXiv:gr-qc/9403028].

[33] T. Jacobson, G. Kang, and R. C. Myers, Phys. Rev. D 49 (1994) 6587; [arXiv:gr-qc/9312023]. Phys. Rev. D 52 (1995) 3518 [arXiv:gr-qc/9503020].

[34] T. Clunan, S. F. Ross and D. J. Smith, Class. Quant. Grav. 21 (2004) 3447 [arXiv:gr-qc/0402044]. See also M. Cvetic, S. Nojiri and S. D. Odintsov, Nucl. Phys. B 628 (2002) 295 [arXiv:hep-th/0112045]. 